\title{
Paper-Based Electrical Respiration Sensor
}

\section{Citation}

Güder, Firat, Alar Ainla, Julia Redston, Bobak Mosadegh, Ana Glavan, T. J. Martin, and George M. Whitesides. 2016. "Paper-Based Electrical Respiration Sensor." Angewandte Chemie International Edition 55 (19) (April 5): 5727-5732. Portico. doi:10.1002/anie.201511805.

\section{Published Version}

doi:10.1002/anie.201511805

\section{Permanent link}

http://nrs.harvard.edu/urn-3:HUL.InstRepos:29946751

\section{Terms of Use}

This article was downloaded from Harvard University's DASH repository, and is made available under the terms and conditions applicable to Open Access Policy Articles, as set forth at http:// nrs.harvard.edu/urn-3:HUL.InstRepos:dash.current.terms-of-use\#OAP

\section{Share Your Story}

The Harvard community has made this article openly available.

Please share how this access benefits you. Submit a story.

Accessibility 


\section{Paper-based Respiration Sensor}

Firat Güder ${ }^{1}$, Alar Ainla ${ }^{1}$, Julia Redston ${ }^{1}$, Bobak Mosadegh ${ }^{1,2}$, Ana Glavan ${ }^{1}$, T.J. Martin ${ }^{1}$, and George M. Whitesides ${ }^{1,2 *}$

${ }^{1}$ Department of Chemistry and Chemical Biology, Harvard University, 12 Oxford Street, Cambridge, MA 02138, USA.

${ }^{2}$ Wyss Institute for Biologically Inspired Engineering, Harvard University, 60 Oxford Street, Cambridge, MA 02138, USA.

(*) Author to whom correspondence should be addressed: gwhitesides@gmwgroup.harvard.edu 


\begin{abstract}
Breathing is one of the primary vital signs used to diagnose the health status of patients; it is related to many common disorders and diseases, ranging from pulmonary and cardiovascular diseases to sleep-related disorders. Current methods of monitoring breathing require cumbersome, inconvenient and often expensive devices; this requirement sets practical limitations on the frequency and duration of measurements. This article describes a paper-based moisture sensor that uses the hygroscopic character of paper (i.e. the ability of paper to adsorb water reversibly from the surrounding environment) to measure patterns and rate of respiration by converting the changes in humidity caused by cycles of inhalation and exhalation to electrical signals. The changing levels of humidity that occur in a cycle causes a corresponding change in the ionic conductivity of the sensor, which can be measured electrically. By combining the paper sensor with conventional electronics, data concerning respiration can be transmitted to a nearby smartphone or tablet computer for post-processing, and subsequently stored on a cloud server, or they can be further analysed by a healthcare professional remotely. This means of sensing provides a new and practical solution to the problem of recording and analysing patterns of breathing.
\end{abstract}




\section{Introduction}

Rate of respiration, together with heart rate, blood pressure and body temperature, are used by healthcare workers to estimate the basic health-status of patients ${ }^{1,2}$. In differentiating between stable and unstable patients, the rate of respiration is a better metric than other vital signs ${ }^{3}$. Abnormalities in the rate and pattern of respiration are a strong predictor of acute events, such as cardiac arrest, or for characterizing illnesses, such as chronic obstructive pulmonary disease (COPD), pneumonia, and asthma ${ }^{4}$.

The respiratory rate of a healthy adult at rest is $12-20$ breaths per minute, corresponding to an exchange of 6-8 litres of air per minute ${ }^{5}$. Fieselmann et. al. reported that a rate of respiration greater than 27 breaths per minute was the most important predictor of cardiac arrest in hospital wards ${ }^{6}$. Cretikos and co-workers observed that, in the general wards, patients suffering from a serious illness, and having a rate of respiration greater than 24 breaths per minute, could be identified as high risk (for intervals up to 24 hours), with a specificity of $95 \%{ }^{3}$. Continuous measurement of the rate and depth of respiration during sleep is also important in diagnosing sleep apnea, a condition estimated to affect 25 million Americans, $80 \%$ of whom remain undiagnosed ${ }^{7-10}$.

In addition to being an important metric for characterizing health, the rate of respiration is a reliable marker for determining the anaerobic threshold (AT), also known as the lactate threshold, in athletes ${ }^{11,12}$. AT is defined as the highest level of exercise that can be maintained without inducing metabolic acidosis ${ }^{13}$; this level is strongly associated with athletic performance.

In most clinical settings, (e.g. the emergency room of a hospital), the rate of respiration is commonly measured by observing the patient from a distance and counting the rising and falling of the chest ${ }^{14}$. Although this method is simple, it is subjective, and provides little, if any, information about the depth of respiration of the patient. It is also challenging and impractical to monitor individual patients visually over extended periods of time ${ }^{15}$. 
Dedicated biomedical instruments accurately monitor the rate of respiration; these instruments usually use the sound, airflow, and movement of the chest ${ }^{16}$, airflow is the most commonly used in clinics. A temperature sensor (e.g. thermistor) is typically used for detection, since exhaled air is usually warmer than the inhaled air, and the cyclical change in temperature can be transduced and correlated to a rate of respiration ${ }^{17}$. This method, however, is prone to errors due to unintentional displacement of the (rigid) sensor during measurement.

Other technologies measure respiratory activity using a pressure sensor ${ }^{18,19}$. In this method, an increase in pressure is registered by the sensor when the subject exhales air. The main drawback of this method is the heavy, non-flexible facemask/mouthpiece or the uncomfortable nasal cannula that the subject must wear. These instruments are also expensive; more than $\$ 150$ without the reader electronics (the components that digitize the recorded analog signal and display the results) and several thousand dollars as a complete system; this cost limits the use of this type of device. Detection of the rate of respiration based on sound using acoustic sensors, such as microphones, has been suggested as an alternative ${ }^{20-22}$. This form of transduction, however, performs poorly when the patient coughs/sneezes, snores or cries (in case of infants) or is in a noisy surrounding.

This article reports an effective, simple, inexpensive sensor for monitoring respiration. The sensor is capable of measuring the rate of respiration of a person by detecting the difference in moisture content of inhaled and exhaled air. The sensor comprises a piece of paper with digitally printed graphite electrodes, and is attached inside a flexible textile procedure mask (commonly used in hospitals). We also designed and fabricated a battery-powered standalone unit that can interface with an internet-enabled tablet computer/smartphone. Our system can display and upload the collected data to the cloud, and thus enable remote access to the results. The paper sensor, electronics, and software, essentially transform a simple textile mask into a functional mask (with internet connectivity), which can measure, analyze, store, and share information concerning the rate and pattern of respiration of individual patients. 


\section{Experimental Methods}

\section{Sensor and Mask Design}

The paper sensor exploits the hygroscopic character of cellulose paper - i.e. its tendency to adsorb water from the environment. For instance, at a relative humidity $(\mathrm{RH})$ of $70 \%$, paper absorbs up to $10 \%$ of its weight in water. Since the ionic conductivity of the paper is proportional to the amount of water on the surface of the cellulose fibers, the changes in moisture content of paper due to breathing can be used to monitor respiration ${ }^{23,24}$.

When breathing out, the human breath is fully humidified (RH 100\%), and therefore, increases the amount of water on the sensor, and thus its ionic conductivity. When breathing in, the amount of water on the surface of the cellulose fibers is reduced because the surrounding atmosphere almost always has a lower RH than the exhaled air. This change in the amount of adsorbed water decreases the ionic conductivity of the sensor (Figure 1). Essentially, the paper sensor transduces variations in the level of moisture of its immediate surrounding to an electrical signal. Using this strategy, we can acquire the rate and pattern of respiration of a person accurately. As the sensor itself measures changes in moisture content between the inspired and expired air, the system requires no calibration.

\section{Materials Fabrication and Characterization}

The paper sensors were fabricated by digitally printing graphite ink (Ercon Graphite Ink 3456) using a ball-point pen and a craft cutter/printer (Graphtec Craft Robo Pro) onto paper ${ }^{25}$. The graphite ink was diluted with a proprietary solvent (Ercon ET160) 55:45 w/w to obtain desired consistency for printing, and the mixture was homogenized using a tip sonicator to create a uniform dispersion. Using this printing technique, large numbers of sensors can be printed with high accuracy (Figure 2). (Other printing techniques such as screen printing or reel-to-reel printing can, of course, also be used for increased throughput). We chose an interdigitated electrode design to increase the area of the electrodes and the signal-to-noise ratio. This design 
also allows rapid access of humidity to the paper. We have also produced sensors with electrodes located on the top and bottom surface of the paper (Figure S1). The power required for this design of the sensor is $250 \mu \mathrm{W}$ at a $\mathrm{RH}$ of $90 \%$, which is similar to the power requirement of the interdigitated design $(175 \mu \mathrm{W})$ at the same RH. Due to slightly higher complexity in manufacturing, we fabricated sensors with the interdigitated design for convenience. The types of paper we used in the experiments were Whatman 3MM Chr and Whatman $1 \mathrm{Chr}$ by GE Healthcare, and copy paper from WB Mason.

We purchased the procedure masks from CVS (CURAD Surgical Facemasks). This brand was chosen purely based on its widespread availability and low cost, but we anticipate that most other brands can also be used successfully.

We characterized the response of the sensors to RH in a system built in-house (see supporting information Figure S2). It consisted of two mass-flow-controllers supplying dry and humidified $\mathrm{N}_{2}$ streams. Both streams of nitrogen were controlled by a computer using LabView software. The electrical measurements were made with a Keithley 2400 source-meter. We designed the electrodes of the paper sensors to fit into the electrical connector in the measurement system such that eight sensors could be measured in parallel.

\section{Electronics and Software}

We designed the reader electronics using off-the-shelf components purchased from Digikey (including the Arduino microcontroller). The printed circuit board (PCB) was designed using Eagle application package and was manufactured by Silver Circuits Sdb. Bhd., Selangot Malaysia. The Bluetooth shield, Kedsum KDF001A, for the Arduino board was purchased separately from Amazon. The housing for the electronics was designed using AutoCAD and printed with a Dimension; Stratasys, Inc 3D printer in acrylonitrile-butadiene-styrene (ABS) thermoplastic polymer.

We analysed the power characteristics of each subsystem as well as the entire device and the paper sensor (Figure S3). The system required a steady supply of $130 \mathrm{~mA}$ when all of the 
components, including the Bluetooth wireless link between the device and the tablet computer, were active at a $5 \mathrm{~V}$ supply voltage. This corresponds to a power requirement of $650 \mathrm{~mW}$. The power specifications can be substantially reduced through further optimization and redesign of the electrical circuit. The paper-sensor had the lowest power requirement among the electronic components used in the system with a peak power consumption of $500 \mu \mathrm{W}(<0.05 \%$ of the entire system).

The Android app was designed and implemented in the Android Studio development environment using Java and XML programming languages. We used a Samsung Galaxy Tab 4 tablet computer for testing.

\section{Human Subjects Research}

We obtained written consent from all subjects who participated in the experiments. The research protocol and all associated materials were approved by the Harvard University, Faculty of Arts and Sciences, Institutional Review Board (IRB) under the reference number IRB15-0949.

\section{Results and Discussion}

\section{Sensitivity of Paper-based Moisture Sensor to Relative Humidity}

The moisture content of paper is a function of the relative humidity of the surrounding environment $^{23,24}$. Figure 3 shows the electrical conductivity of the paper-based moisture sensors, fabricated from different types of paper, over a range of $\mathrm{RH}(0-90 \% \mathrm{RH})$. We performed these electrical measurements by applying a 25V DC potential across the graphite electrodes and measuring the resulting current. We chose $25 \mathrm{~V}$ to obtain a high signal-to-noise ratio. Currents less than $1 \mathrm{nA}$ had a poor signal-to-noise ratio and were not used.

We used three different kinds of papers for the fabrication of the paper sensors: i)

Whatman $1 \mathrm{Chr}$, ii) Whatman 3MM Chr and, iii) copy paper. Both Whatman $1 \mathrm{Chr}$ and Whatman 
3MM Chr papers are made of pure cotton cellulose fiber with a basis weight of $87 \mathrm{~g} / \mathrm{m}^{2}$ and 185 $\mathrm{g} / \mathrm{m}^{2}$. (The basis weight of paper is defined as the weight of paper per unit area) ${ }^{26}$. The standard copy paper had a basis weight of $80 \mathrm{~g} / \mathrm{m}^{2}$. None of the sensors fabricated from these papers generated a readable current below an $\mathrm{RH}$ of $20 \%$. Whatman $1 \mathrm{Chr}$ had the lowest sensitivity, and did not produce a distinguishable signal below an $\mathrm{RH}$ of $65 \%$. Whatman $3 \mathrm{MM} \mathrm{Chr}$ was significantly more sensitive than Whatman $1 \mathrm{Chr}$, and detected all levels of $\mathrm{RH}>30 \%$. The difference in sensitivity between sensors fabricated with Whatman 3MM Chr and Whatman 1 Chr may be due to their basis weight. A larger area of cellulose fibers in a given area of paper would create a greater number of electrically conductive pathways at a given level of $\mathrm{RH}$, reduce the overall resistivity, and increase the sensitivity. The copy paper showed the highest sensitivity. The high sensitivity of copy paper may be related to various sizing components, particularly clay, added in the papermaking process. Clay is added to create a smooth surface for increased printability. The added clay increased both the concentration of ions in the paper substrate and the hydrophilicity of the system, and thus increase conductivity ${ }^{27}$. The high sensitivity of the copy paper, however, resulted in 10 times higher power consumption compared to Whatman 3MM Chr. We, therefore, used Whatman 3MM Chr for the remainder of the experiments.

The sensor is a simple two-electrode electrochemical cell, in which water (from atmosphere or breath) is electrolyzed on the application of an electrical potential between the electrodes. The corresponding oxidation and reduction reactions of water on the surface of the two graphite electrodes produce a measurable electrical current. We expect the only electrolysis products to be small quantities of $\mathrm{O}_{2}$ and $\mathrm{H}_{2}$.

We observed only a weak dependence of the output current of the paper sensor on the temperature (between $22^{\circ} \mathrm{C}$ and $40{ }^{\circ} \mathrm{C}$; Figure S4). We attribute this small difference to an increase in the mobility of ions at elevated temperatures, and thus an increase in conductivity ${ }^{24}$. The observed difference, however, was small enough (20\% at RH 90\%) to neglect. 
We also verified that the paper sensor is not sensitive to $\mathrm{CO}_{2}$ (see Figure S5) up to a mole fraction in the gas flowing through the sensor of $25 \%$. We presume this insensitivity is due to the low solubility of $\mathrm{CO}_{2}$ in water $(<0.5 \mathrm{~g} / \mathrm{L}$, room temperature, $1 \mathrm{~atm}){ }^{28}$. The paper sensor is, therefore, primarily sensitive to changes in the amount of moisture present in paper, and is not affected by the concentration of $\mathrm{CO}_{2}$ in human breath $(<5 \%)$.

The electrical conductivity of paper can be modified by the addition of ionic species, which increase the concentration of charge carriers in paper, and thus, increase transport of charges between the electrodes. Widely available water soluble ionic compounds, such as salts, therefore, represent a simple, yet effective method to fine-tune the electrical properties of the paper sensor (Figure S6 and S7).

\section{Recording and Analysis of Respiratory Signals}

We placed pure cellulose paper-based moisture sensors, with no added salts, in textile procedure masks to demonstrate their use with healthy adults (seven members of our research team - Figure 4a).

We designed and fabricated a simple device for data acquisition using off-the-shelf electronic components (Figure $4 \mathrm{~b}$ and $\mathrm{c}$ ). This instrument generated a $25 \mathrm{~V}$ DC potential from a $5 \mathrm{~V}$ DC power source, and applied the voltage across the electrodes of the paper sensor. The unit amplified and digitized the resulting electrical current and transmitted the data to a tablet computer (Figure 4d) over a wireless Bluetooth communication link. The custom-built Android application, with a minimally complex design, displayed the incoming data and ran simple analytics (e.g. Fourier transformations). The software could also save the collected results to a text file, which could be uploaded to the cloud, or emailed to a third party for off-site analysis. This feature would be particularly useful for individuals who might monitor their respiration at home, and wish to share their results remotely.

The Android application also has the option to apply finite-impulse-response (FIR) and

infinite-impulse-response (IIR) digital filtering algorithms to the acquired signal ${ }^{29}$. Digital filters 
are commonly used for the separation of combined signals and the restoration of distorted (i.e. noisy) signals. Since the system we described can be used as a research tool, the user has the flexibility to design custom digital filters (e.g. low-pass, high-pass, band-pass, band-stop) to extract other metrics from the recorded pattern of respiration. The filter coefficients can be stored as a text file on the tablet computer and imported when the application is launched. Both the filtered and the raw data are recorded as a text file for later analysis. By implementing additional compressed sensing / sparse sampling algorithms, the size of the files containing the collected data can be made significantly smaller, to enable use of networks with low speeds. This capability is required in the developing world ${ }^{30,31}$.

The functional textile mask was able to track the respiratory activity of the subjects successfully up to 15 minutes at room temperature (Figure 5a and Figure S9 - S14) while at rest (i.e. sitting in a chair); the duration of the experiment was limited by the specifications of the IRB. There is a significant initial drift in the output of the paper sensor. The drift probably reflects the large difference in drying and humidifying cycles during breathing, and reaches equilibrium in a few minutes. Since, however, we are primarily interested in measuring the intervals between the signal maxima, the change in the absolute level of signal caused by the drift is not relevant. Figure $5 \mathrm{~b}$ displays different breathing patterns acquired during a representative experiment with a single subject. The area marked with (1) is a normal breathing pattern (which consists of periodic breathing cycles of similar magnitude) followed by a deep breath marked as (2). During this period, the subject had a rate of respiration of 14 breaths per minute. A pause in breathing was registered as a drop in the recorded output current (3). A mixture of deep, slow, fast, shallow breathing patterns could also be accurately monitored during the experiment and marked as (4) in Figure 5b. The device responded seamlessly to the transition between periods of fast, shallow and normal breathing (Figure 5c). The system was also able to acquire and transmit all of the collected data to the tablet computer.

Figure 6 shows one of the trials (see Figure S15 - S19 for data from the rest of the trials) in which a subject took a short walk inside the building to test two performance factors of the 
system: i) noise levels in the collected data during movement, and ii) system performance during light exercise (i.e. walking around the hallways) and vigorous exercise (i.e. high tempo climbing of four flights of stairs in the building). The recorded data had little observable noise during both light exercise (Figure 6b) and vigorous exercise (Figure 6c); the rate of respiration could easily be detected by counting the number of peaks. The subject registered a rate of respiration of 12 breaths/min during light exercise, and 22 breaths/min during more vigorous exercise. (The breath counts were calculated manually from the collected data). Interestingly, some of the subjects (Figure S8) decreased their rate of respiration when transitioning from light exercise (24 breaths $/ \mathrm{min}$ ) to vigorous exercise ( 16 breaths/min). These subjects compensated for the decrease in rate of respiration by taking deeper breaths (Figure S6b and S6c). Figure 7a summarizes the rate of respiration of each subject during light and vigorous exercise.

To provide an additional metric (one related to the respired volume) for the respiration of subjects, we analysed the amplitude of the signal generated by the sensor. The amplitude of the signal correlates to the depth of breathing by the subject. When a subject transitions from light exercise to vigorous exercise, two parameters related to respiration may change: i) the rate of respiration (RR), which can be calculated by counting the number of peaks in the captured cyclic signal, and ii) the depth of respiration (DR), which we assume to correlate (undoubtedly nonlinear with) the peak-to-peak amplitude of the train of breaths captured by the device. We defined an empirical metric, the Breathing Index (BI) by Eq. 1 (see Figure S20 for more details on BI).

$$
B I=\left(\frac{R R_{V i g o r o u s}}{R R_{\text {Light }}}\right) \times\left(\frac{D R_{\text {Vigorous }}}{D R_{\text {Light }}}\right)
$$

Here, $R R_{\text {vigorous }}$ is the rate of respiration during vigorous exercise, $R R_{\text {Light }}$ is the rate of respiration during light exercise, $\mathrm{DR}_{V_{i g} \text { orous }}$ is the peak-to-peak amplitude of the signal during vigorous exercise, and $\mathrm{DR}_{\mathrm{Light}}$, is the peak-to-peak amplitude of the signal during light exercise. This approach, leads to the obvious conclusion that all of the subjects exchanged a larger volume of air during vigorous exercise (BI > 1) than they did during light exercise (Figure 7b), a 
conclusion that cannot be derived solely from the rate of respiration. Our analysis indicated that Subject \#5 is more fit than Subject \#1, since Subject \#5 has the lowest breathing index among all participants in the experiment.

Overall, the paper sensor, electronics and the mobile app, performed without failure or interruption of data during the experiments, and thus has the potential to be used for monitoring breathing at rest and during physical activity. An inexperienced individual could learn how to read and interpret these graphs within a few minutes. Filtering out the drifting baseline using digital filters would further simplify the process of manual interpretation.

\section{Conclusions}

The paper-based electrical respiration sensor is a sufficiently low-cost $(\$ 0.005$ for materials for the paper chip and $\$ 1.50$ for the mask) that it can be considered for single-use applications. It enables continuous monitoring of the respiratory activity of a number of populations (e.g., patients, athletes, research subjects, smokers, perhaps children). The information is obviously less accurate than that available in a pulmonology laboratory, but is more convenient and perhaps more useful in routine and at-home monitoring. We believe this sensor will be (at least initially) most immediately useful in characterizing sleep apnea. In addition, we believe athletes, or others to whom fitness is important, might use this sensor to monitor breathing to track changes in their performance. The fabrication procedure for the sensor is simple; it requires only a digital printer and a conductive ink (graphite ink in our case). We were able to print more than 40 sensors in 15 minutes with this method, in our lab, but printing could easily be adopted to much higher volume methods (e.g., screen printing, reel-to-reel printing). Since we compare changes in conductivity for inhalation and exhalation during each breathing cycle, we eliminate the need to calibrate for drift, or to remove drift instrumentally: the sensor is self-referenced. The inevitable drift in the mean water content of the paper - a drift that 
is usually a disadvantage of paper as a component of sensors, and often a disqualifying characteristic - is irrelevant here.

The paper-based respiration sensor, combined with conventional electronics, is capable of collecting and sending respiration data to a tablet computer or a smartphone using wireless connectivity. The custom-built Android app running on the tablet computer/smartphone has the ability to run simple analytics (e.g. Fourier transforms) on the incoming data stream, and apply digital filtering algorithms for signal processing. Both the raw and the filtered data can be uploaded to the cloud and shared with a healthcare professional with the click of a button, thus eliminating unnecessary visits to the clinic.

The system has three limitations: i) The power requirement for electronics is too high for intervals of use up to a full day on a single charge of the battery (although it should be adequate for one complete night of sleep: the current design can run for $9 \mathrm{~h}$ on a single charge using a 18650 type lithium ion battery with a nominal capacity of $2600 \mathrm{mAh}$ ); the data acquisition system requires daily charging of its batteries. Full day or week monitoring would require more battery capacity. ii) The electrodes of the sensor are prone to cracking if the paper is folded, but folding should not occur during regular use of the sensor. iii) We expect the paper sensor may not work efficiently, or at all, at temperatures $<0{ }^{\circ} \mathrm{C}$ due to freezing of the moisture and build-up of ice inside the paper. We expect that the performance of the sensor would also be reduced on a day with RH $100 \%$ at $37^{\circ} \mathrm{C}$, however, such conditions are not common (in Singapore, RH $100 \%$ can occur during prolonged periods of rainfall but the temperatures never exceed $36^{\circ} \mathrm{C}^{32}$ ).

The paper sensor has a short (on the time-scale required to measure breathing) response time. We were able to capture rates of respiration up to 60 breaths per minute without loss of precision. This performance is adequate for most applications.

The operation of the system is sufficiently simple that inexperienced, first-time users can be trained in a matter of a few minutes; all they have to do is to put on the mask, run the Android app and click on "start" in order to monitor and record respiration. 


\section{Acknowledgements}

This work was funded by DTRA (Contract: HDTRA1-14-C-0037). The research of F.G. is sponsored by the German Research Foundation - DFG (Contract: GU 1468/1-1). A.A. thanks the Swedish Research Council for financial support. We would like to thank Mr. Jim McArthur and the Electronic Instrument Design Lab (Department of Physics, Harvard University) for assistance in fabrication of electronics. We would also like to thank Dr. Victoria Campbell, Dr. Chien-Chung Wang, Dr. K.C. Liao, Dr. Nicolas Fulleringer, Dr. Sergey Semenov, Mr. Philipp Rothemund, Dr. Mahiar Hamedi, Dr. Baris Unal and Dr. Robert Kacmarek for fruitful discussions.

\section{Author Contributions}

F.G led the work and developed the initial ideas, designed, fabricated and characterized the paper sensors, contributed to hardware and software development, collected the human data and wrote the manuscript. A.A. contributed to hardware and software development and was a key contributor to the work. A.A. also contributed to writing. J.R. helped with software development. B.M. was involved in the development of the initial ideas and was a significant contributor to the writing of the manuscript. A.G. performed the initial experiments on sensor fabrication and also contributed to writing. T.J.M. contributed to the IRB approval process and writing. G.M.W contributed to the development of the ideas, and the writing of the manuscript.

\section{Competing Financial Interests}

Authors declare no competing financial interest. 


\section{References}

1 Parkes, R. Rate of respiration: the forgotten vital sign. Emerg. Nurse 19, $12-17$ (2011).

2 Edmonds, Z. V., Mower, W. R., Lovato, L. M. \& Lomeli, R. The reliability of vital sign measurements. Ann. Emerg. Med. 39, 233-237 (2002).

3 Cretikos, M. A. et al. Respiratory rate: the neglected vital sign. Med. J. Aust. 188, 657 (2008).

4 Woollard, M. \& Greaves, I. 4 Shortness of breath. Emerg. Med. J. 21, 341-350 (2004).

5 Ganong, W. F. \& Barrett, K. E. Review of medical physiology. Vol. 21 (McGraw-Hill Medical New York, 2005).

6 Fieselmann, J. F., Hendryx, M. S., Helms, C. M. \& Wakefield, D. S. Respiratory rate predicts cardiopulmonary arrest for internal medicine inpatients. J. Gen. Intern. Med. 8, 354-360 (1993).

7 Noonan, D. The Not So Silent Epidemic. Sci. Am. 312, 27-28 (2015).

8 Peppard, P. E. et al. Increased prevalence of sleep-disordered breathing in adults. Am. J. Epidemiol. 177, 1006-1014 (2013).

9 Gami, A. S., Howard, D. E., Olson, E. J. \& Somers, V. K. Day-night pattern of sudden death in obstructive sleep apnea. N. Engl. J. Med. 352, 1206-1214 (2005).

10 Strollo Jr, P. J. et al. Upper-airway stimulation for obstructive sleep apnea. N. Engl. J. Med. 370, 139-149 (2014).

11 Carey, D. G., Pliego, G. \& Raymond, R. How endurance athletes breathe during incremental exercise to fatigue: Interaction of tidal volume and frequency. J. Exerc. Physiol 11, 44-51 (2008).

12 Carey, D. G., Schwarz, L. A., Pliego, G. J. \& Raymond, R. L. Respiratory rate is a valid and reliable marker for the anaerobic threshold: implications for measuring change in fitness. $J$. Sports Sci. Med. 4, 482 (2005).

13 George, R. B. Chest Medicine: Essentials of Pulmonary and Critical Care Medicine. (Lippincott Williams \& Wilkins, 2005).

14 Karlen, W. et al. Improving the accuracy and efficiency of respiratory rate measurements in children using mobile devices. PLoS One 6, 99266 (2014).

15 Folke, M., Cernerud, L., Ekström, M. \& Hök, B. Critical review of non-invasive respiratory monitoring in medical care. Med. Biol. Eng. Comput. 41, 377-383 (2003).

16 AL-Khalidi, F. Q., Saatchi, R., Burke, D., Elphick, H. \& Tan, S. Respiration rate monitoring methods: A review. Pediatr. Pulmonol. 46, 523-529 (2011).

17 Storck, K., Karlsson, M., Ask, P. \& Loyd, D. Heat transfer evaluation of the nasal thermistor technique. Biomedical Engineering, IEEE Transactions on 43, 1187-1191 (1996). 
18 Norman, R. G., Ahmed, M. M., Walsleben, J. A. \& Rapoport, D. M. Detection of respiratory events during NPSG: nasal cannula/pressure sensor versus thermistor. Sleep 20, 11751184 (1997).

19 Lee-Chiong, T. L. Sleep: a comprehensive handbook. (Wiley Online Library, 2006).

20 Autet, L. et al. Evaluation of acoustic respiration rate monitoring after extubation in intensive care unit patients. Br. J. Anaesth. 113, 195-197 (2014).

21 Guechi, Y. et al. Assessment of noninvasive acoustic respiration rate monitoring in patients admitted to an Emergency Department for drug or alcoholic poisoning. J. Clin. Monit. Comput., 1-6 (Available Online DOI: 10.1007/s10877-10015-19658-y), doi:10.1007/s10877015-9658-y (2015).

22 Jin, H. et al. Acoustic Analysis of Snoring in the Diagnosis of Obstructive Sleep Apnea Syndrome: A Call for More Rigorous Studies. J. Clin. Sleep Med. 11, 765-771 (2015).

23 Tobjörk, D. \& Österbacka, R. Paper electronics. Adv. Mater. 23, 1935-1961 (2011).

24 Mark, R. E. \& Borch, J. Handbook of physical testing of paper. Vol. 1 (CRC Press, 2001).

25 Glavan, A. C. et al. Folding Analytical Devices for Electrochemical ELISA in Hydrophobic RH Paper. Anal. Chem. 86, 11999-12007 (2014).

26 Hutten, I. M. Handbook of nonwoven media. 245 (Elsevier, 2007).

27 Roe, R. B. Clays for use in paper-making. J. Am. Ceram. Soc. 2 (1919).

28 Dodds, W., Stutzman, L. \& Sollami, B. Carbon dioxide solubility in water. Industrial \& Engineering Chemistry Chemical \& Engineering Data Series 1, 92-95 (1956).

29 Smith, S. W. The Scientist and Engineer's Guide to Digital Signal Processing. 261-342 (California Technical Publishing, 1997).

30 Marvasti, F. et al. A unified approach to sparse signal processing. EURASIP J. Adv. Sig. Proc. 2012, 44 (2012).

31 Holland, D. J. \& Gladden, L. F. Less is more: how compressed sensing is transforming metrology in chemistry. Angew. Chem. Int. Ed. 53, 13330-13340 (2014).

32 Meteorological Service Singapore, Climate of Singapore, http://www.weather.gov.sg/climate-climate-of-singapore 

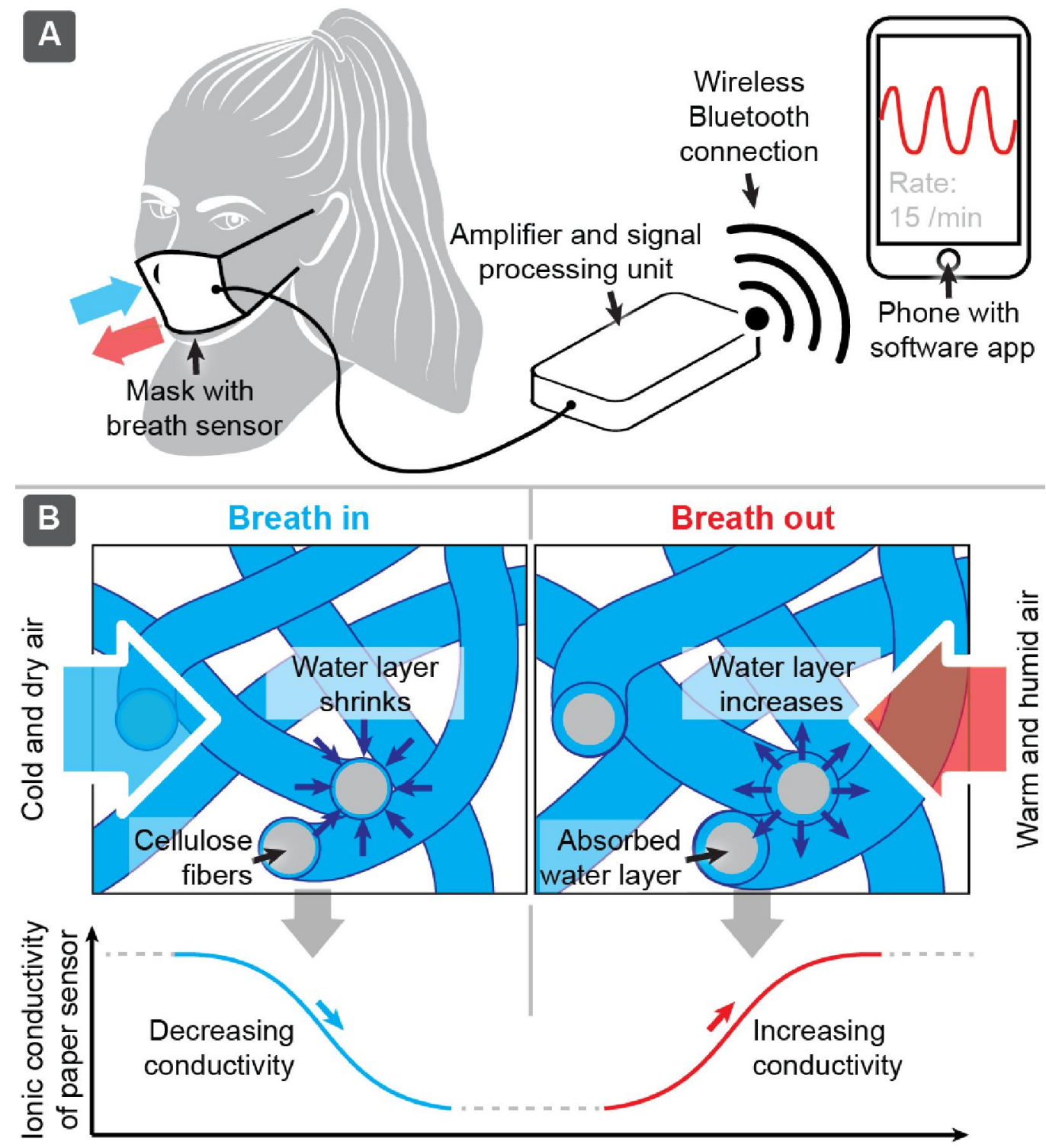

Figure 1. (A) Schematic illustration of the facemask for respiration monitoring with the embedded paper-based sensor and electronics. (B) Mechanism of operation of the paper-based electrical respiration sensor. 


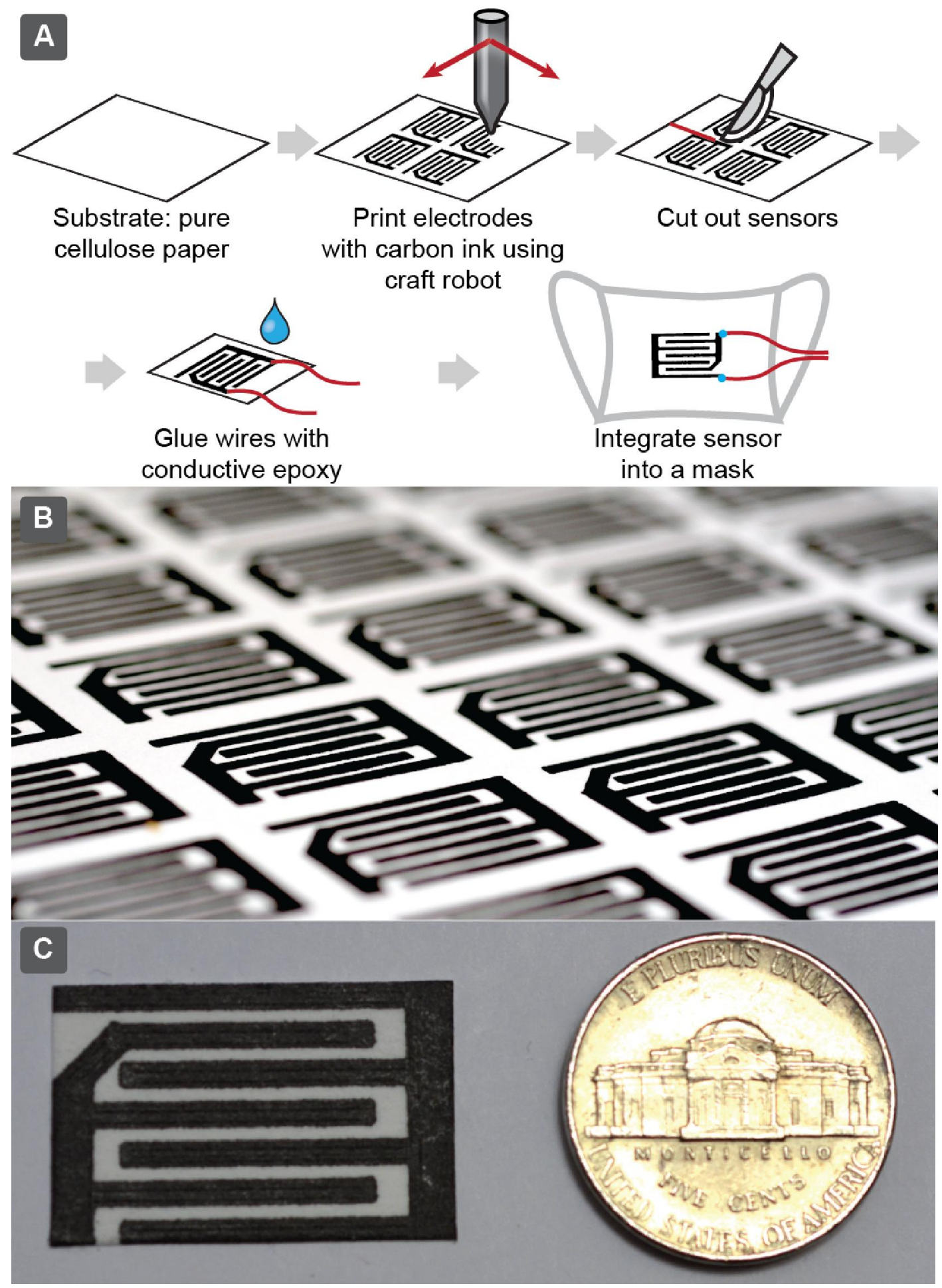

Figure 2. (A) Schematic illustration of the fabrication of digitally printed paper sensors with graphite ink. (B) Image of an array of sensors; (C) An individual paper-based sensor next to a US Nickel. 


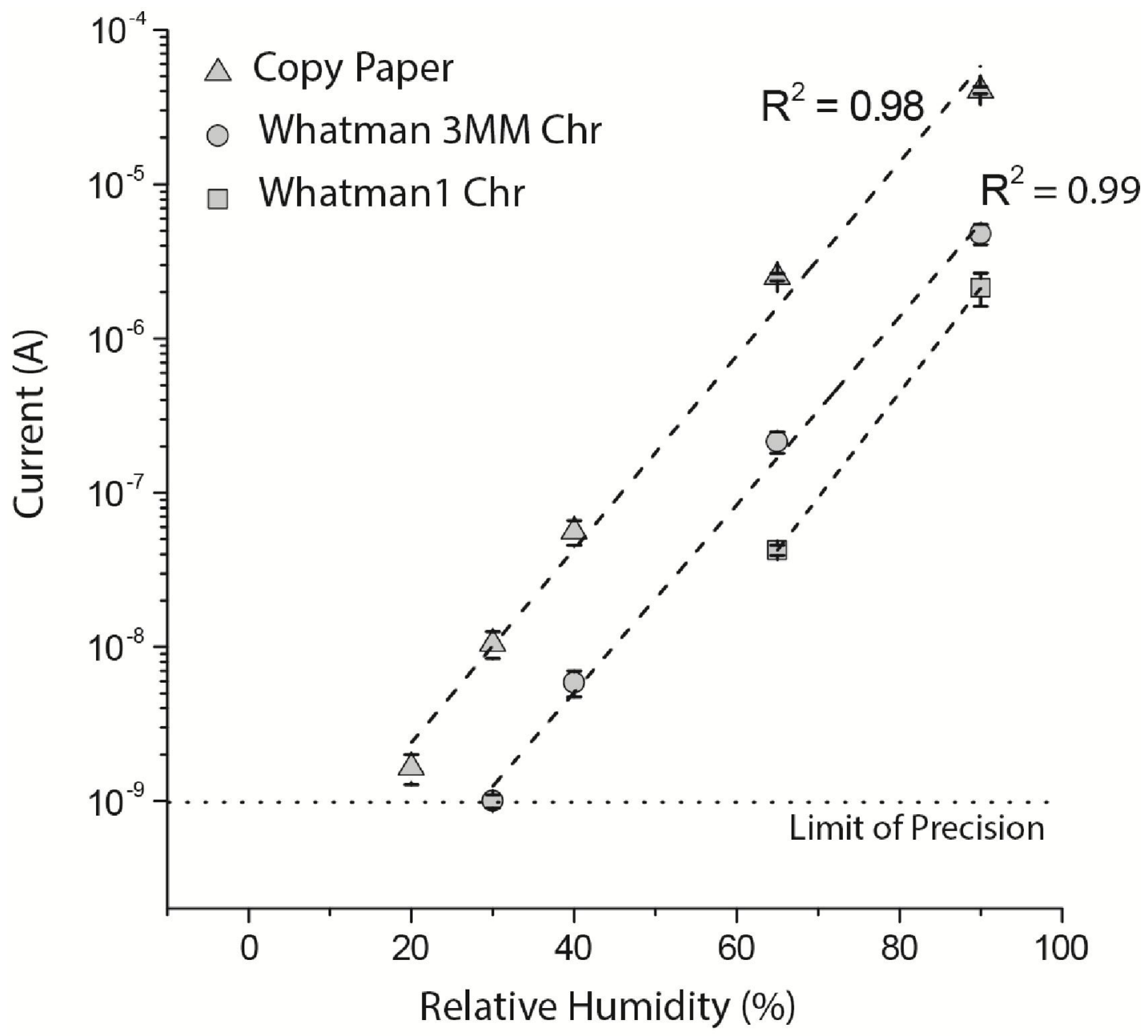

Figure 3. Electrical characterization of the paper-based moisture sensor, fabricated on different paper substrates. The error bars represent standard deviations (SD) for $\mathrm{N}=7$ 


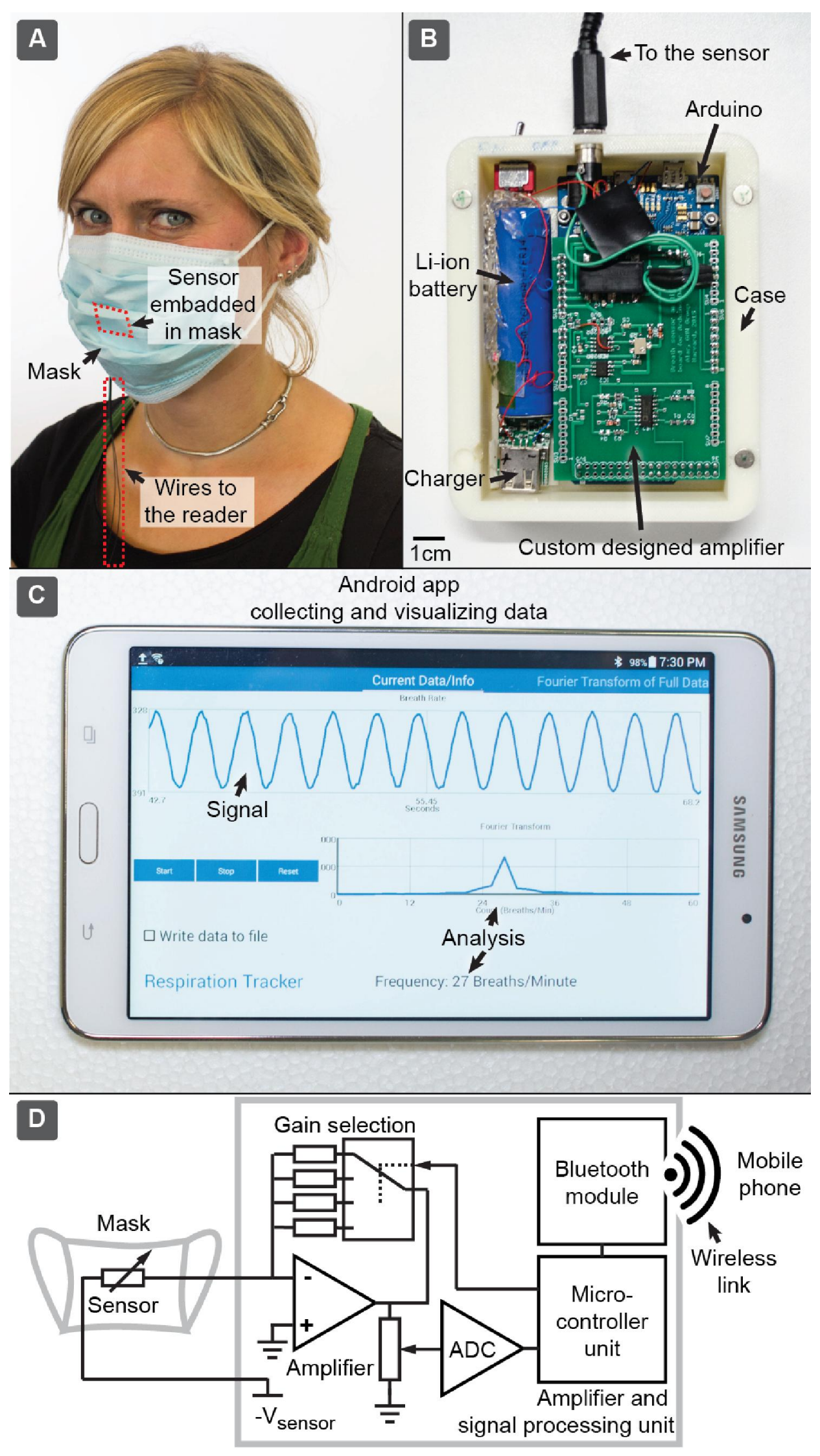


Figure 4. (A) The facemask with the embedded paper-based sensor. (B) Photograph of the data acquisition electronics with Li-ion batteries, Arduino microcontroller board, custom designed amplifier board, and 3D printed casing. (C) Simplified circuit diagram of the electronics. (D) Photograph of tablet computer running the Android app, which can display and analyse the incoming data stream from the data acquisition electronics. 

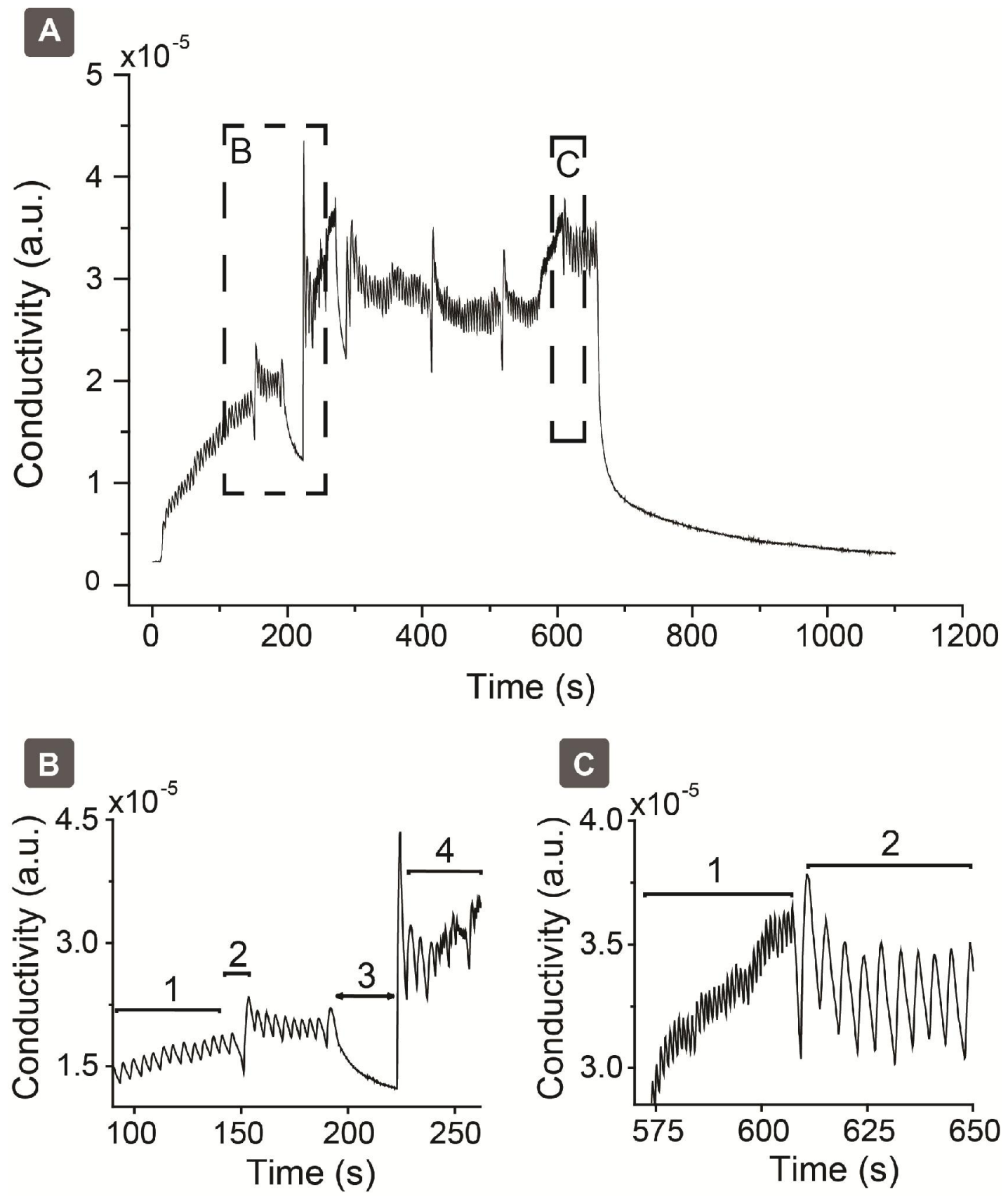

Figure 5. (A) The resting respiratory activity of a subject recorded using the functional facemask. We asked the subject to breathe normally, take deep breaths, pause and breathe randomly during the experiment. (B) Breathing patterns recorded while: 1. breathing normally, 2. taking a deep breath, 3. paused and, 4. random breathing. (C) Plot showing the seamless transition between fast and shallow breathing (1) and normal breathing (2) 

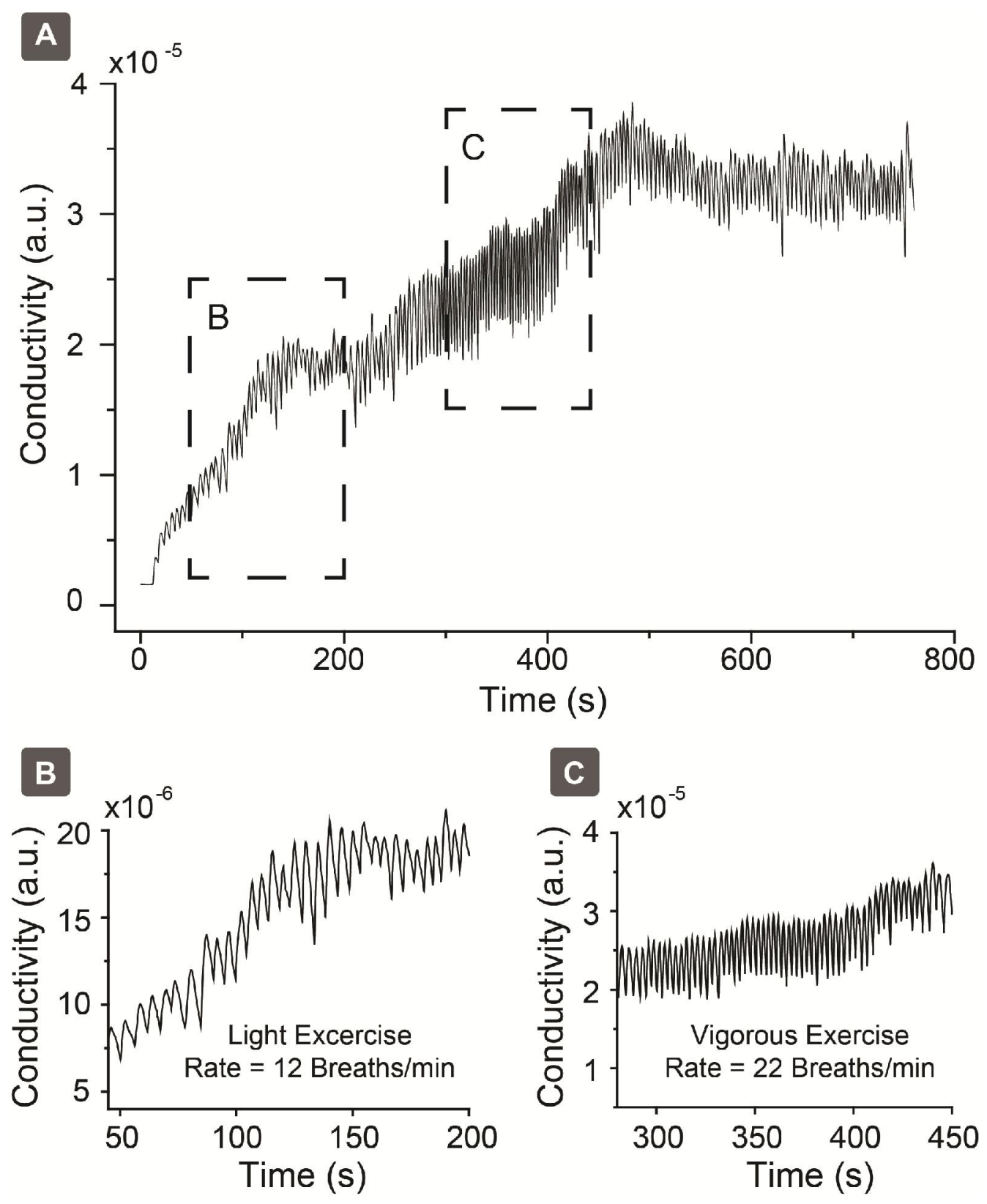

Figure 6. (A) Respiratory signal during light (B) and vigorous (C) exercise. The subject in this experiment had an increase in the rate of respiration from 12 breaths/min during light exercise to 22 breaths/min during vigorous exercise. 


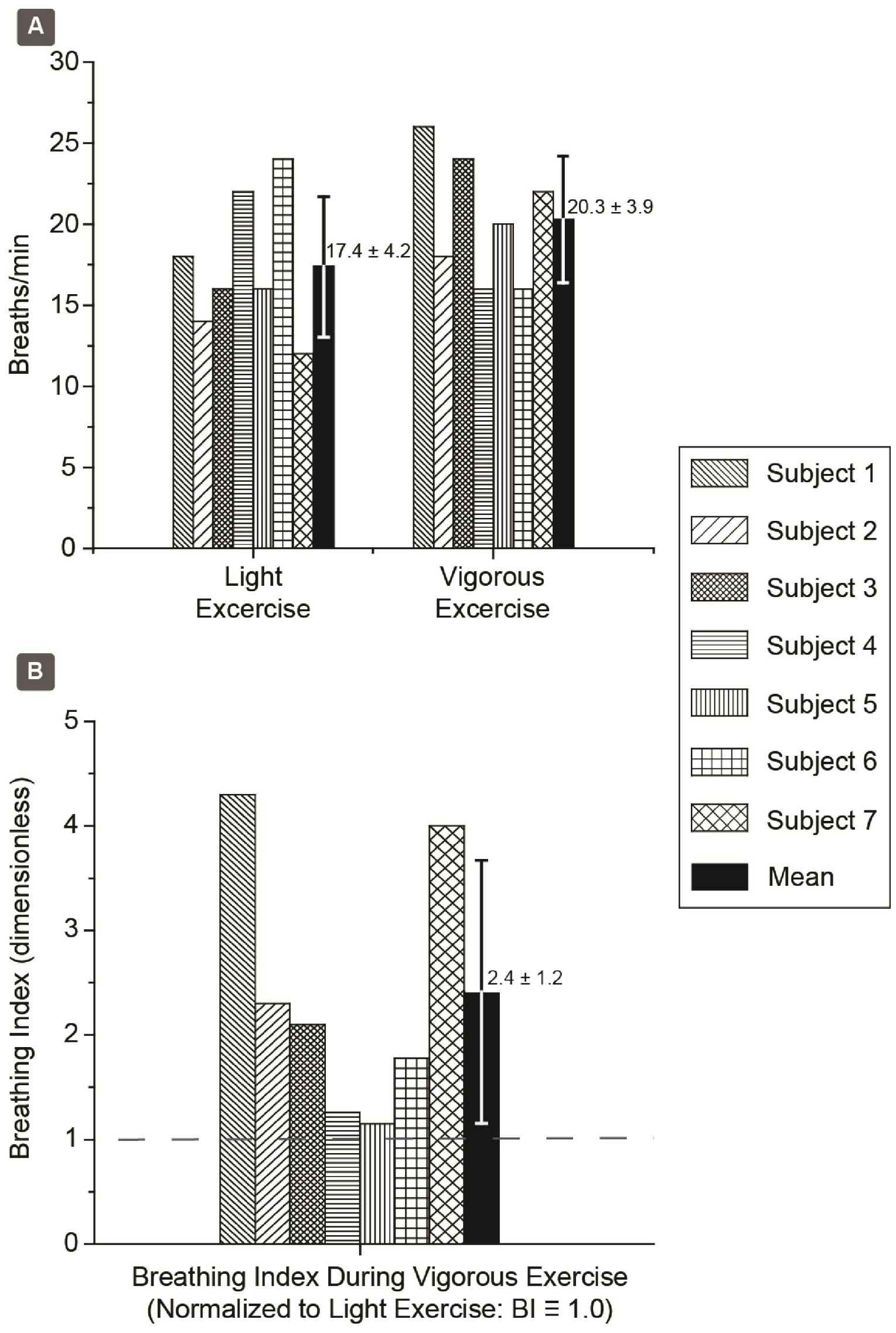


Figure 7. Summary of the rate of respiration (A) of each subject during light and vigorous exercise. (B) The Breathing Index of each subject during light and vigorous exercise. Although some subjects exhibited an increasing or decreasing rate of respiration when transitioning from light exercise to vigorous exercise, all of the subjects respired a higher volume of air during vigorous exercise than light exercise indicated by a $\mathrm{BI}>1$. (Error bars represent standard deviations for $\mathrm{N}=7$ ) 


\section{Supporting Information}

\section{Paper-based Respiration Sensor}

Firat Güder ${ }^{1}$, Alar Ainla ${ }^{1}$, Julia Redston ${ }^{1}$, Bobak Mosadegh ${ }^{1,2}$, Ana Glavan ${ }^{1}$, T.J. Martin, and George M. Whitesides ${ }^{1,2 *}$ 
$\mathbf{a}$

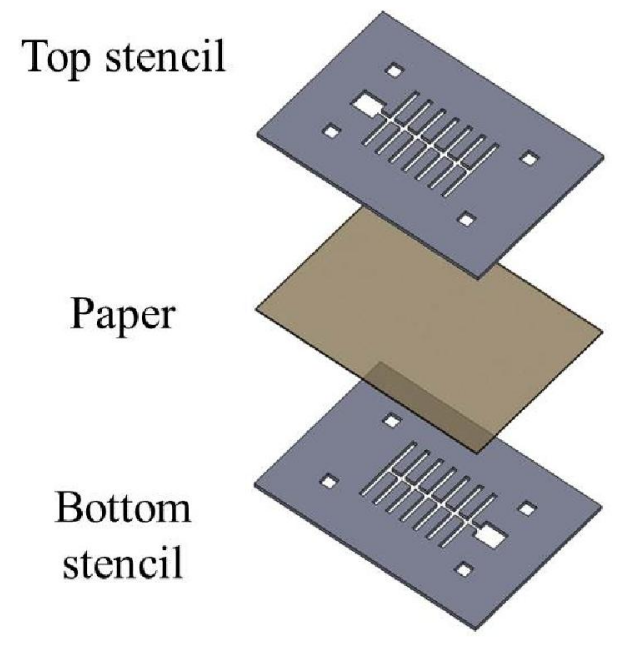

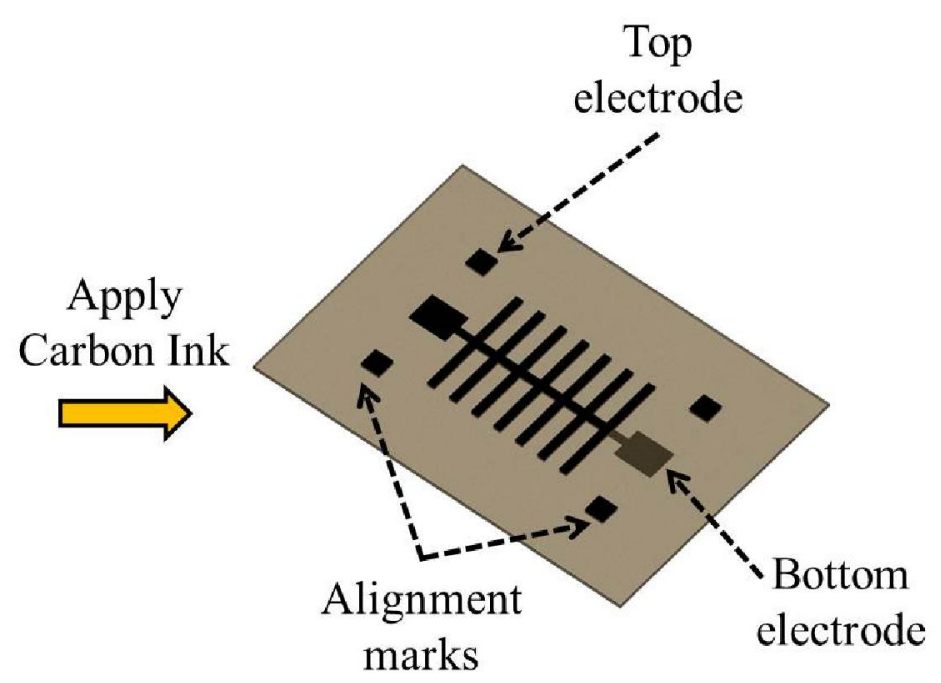

b

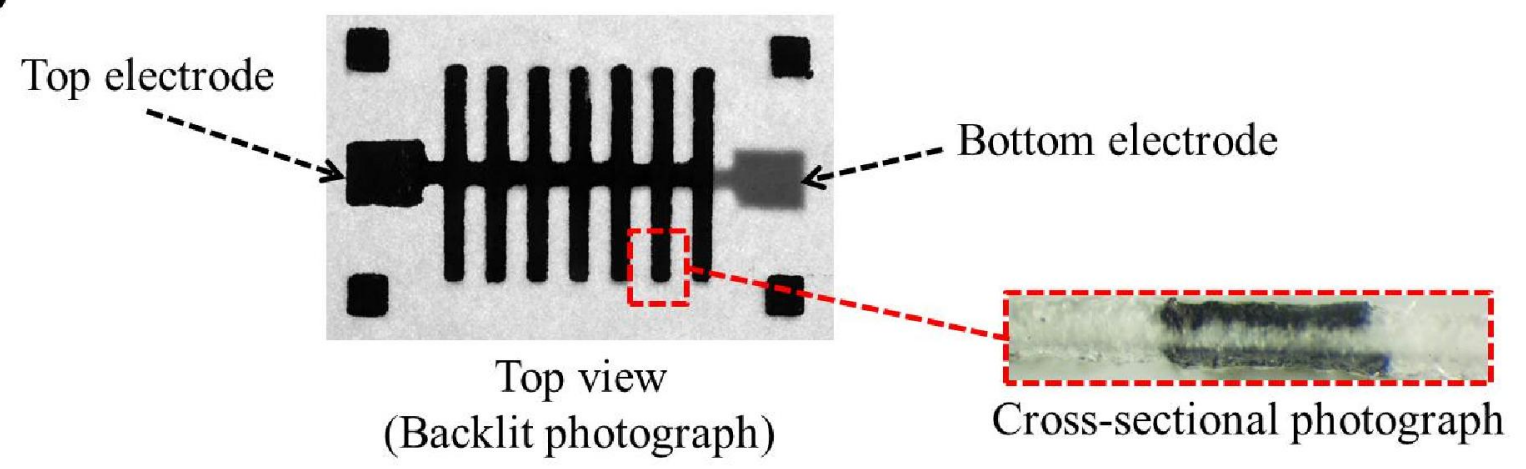

Figure S1. (A) Fabrication procedure of the paper sensor with aligned electrodes printed on both sides of paper (top and bottom) (B) Light photograph of an actual device. The light source is behind the device, showing the aligned electrodes at both the front and back of the paper. 


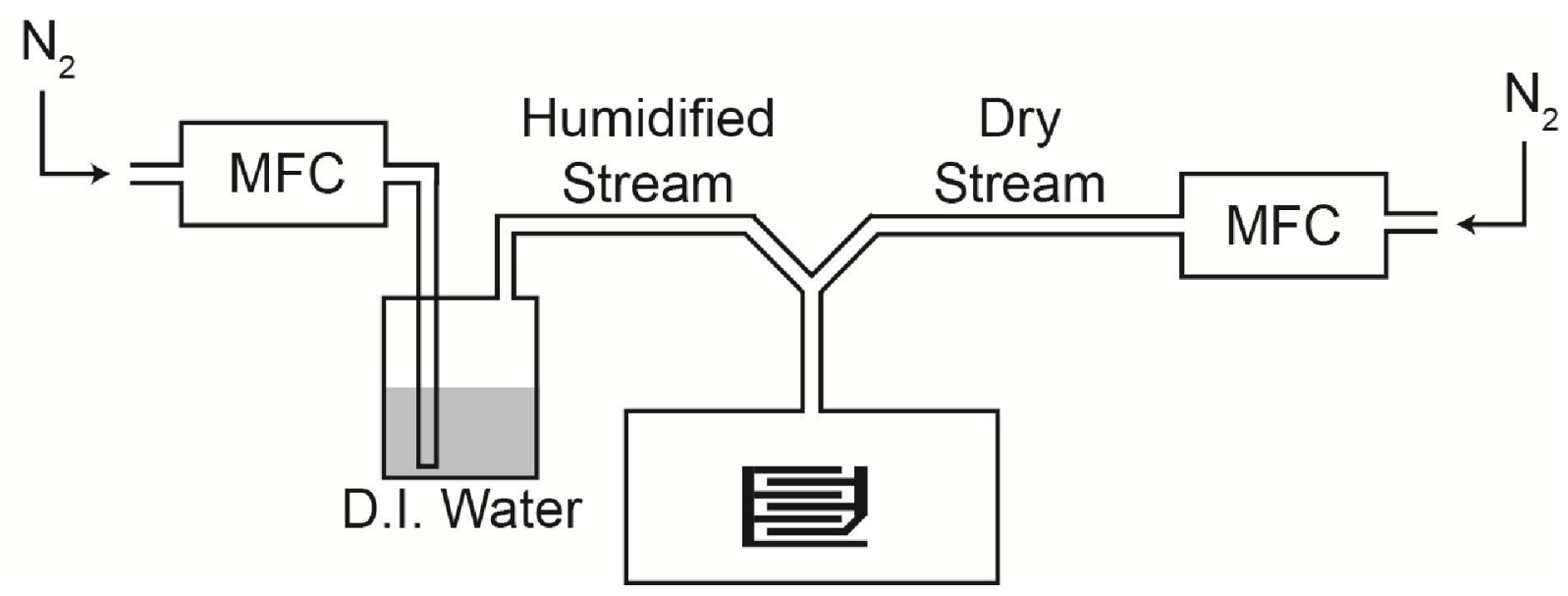

Figure S2. Schematic view of the system constructed to characterize the paper-based sensor. We used two mass flow controllers - MFC - to mix flows of humidified nitrogen and dry nitrogen to control the RH inside the measurement chamber. The humidified stream was created by bubbling nitrogen through a bottle containing deionized water (D.I. water) 


\begin{tabular}{lrrrr}
\hline Part & Voltage (V) & \multicolumn{1}{c}{ Current (mA) } & Power (mW) & Percentage[\%) \\
\hline Battery (2x Li-ion cell). TOTAL & 7.8 & 130 & 1014 & 100 \\
Sensor (dry/min) & 25 & $1.30 E-03$ & 0.0325 & 0.003 \\
Sensor (humid/max) & 25 & $2.00 E-02$ & 0.5 & 0.049 \\
Bluetooth module & 5 & 25 & 125 & 12 \\
Amplifier board & 5 & 36 & 180 & 18 \\
Microcontroller board (Arduino) & 3.3 & 69 & 227.7 & 22 \\
Voltage conversion losses & & & 481.3 & 47 \\
\hline
\end{tabular}

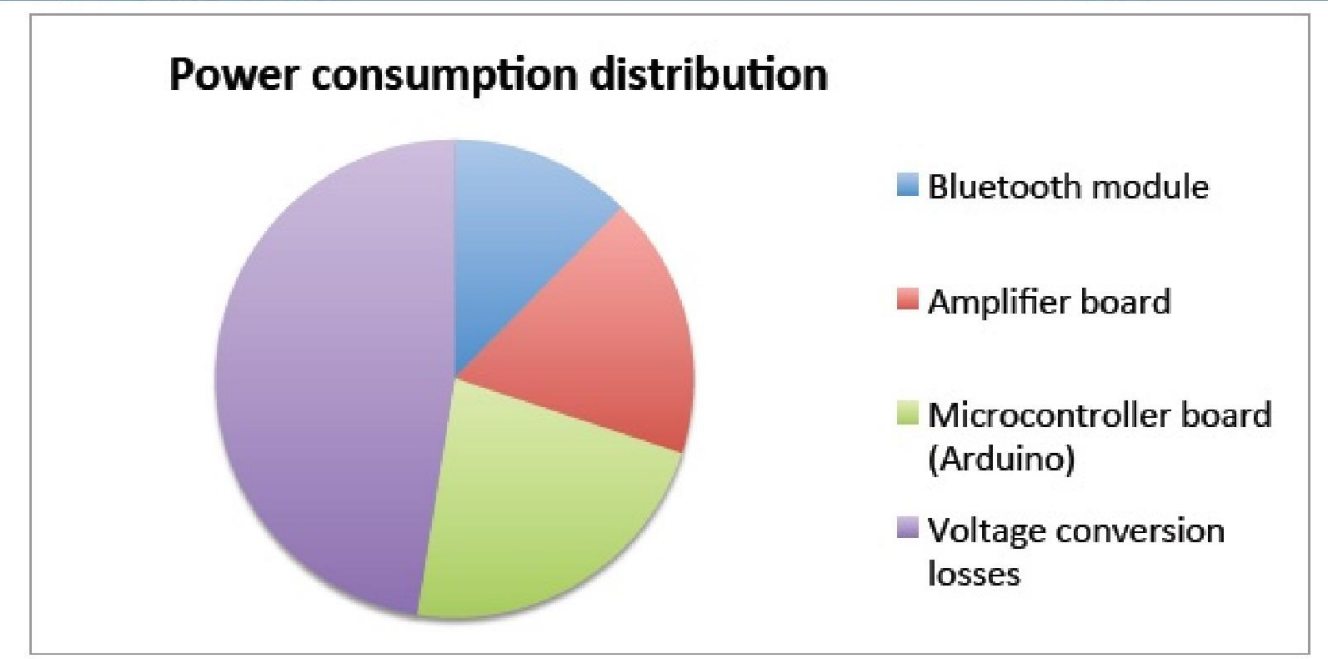

Figure S3. Power consumption distribution of the data acquisition electronics. The current design requires a steady supply of $130 \mathrm{~mA}$. Our design has large voltage conversion losses and which can be eliminated by optimizing the system for power, especially the step-up DC-DC converter and voltage regulator circuitry. Reducing clock speed for the microcontroller board would also reduce power consumption 


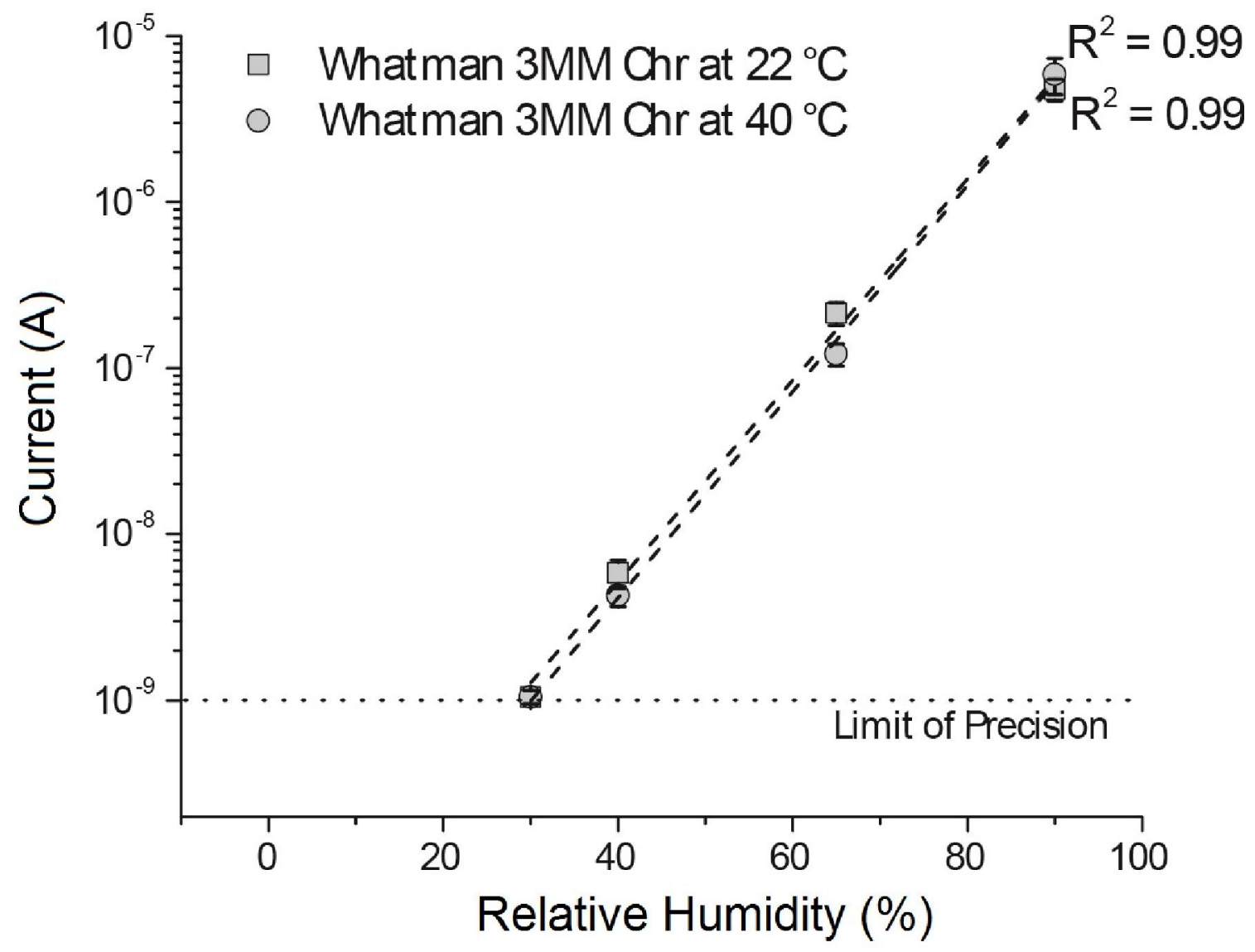

Figure S4. Characterization of paper-based moisture sensors at $22^{\circ} \mathrm{C}$ and $40{ }^{\circ} \mathrm{C}$. The error bars represent standard deviation $(\mathrm{SD})$ 


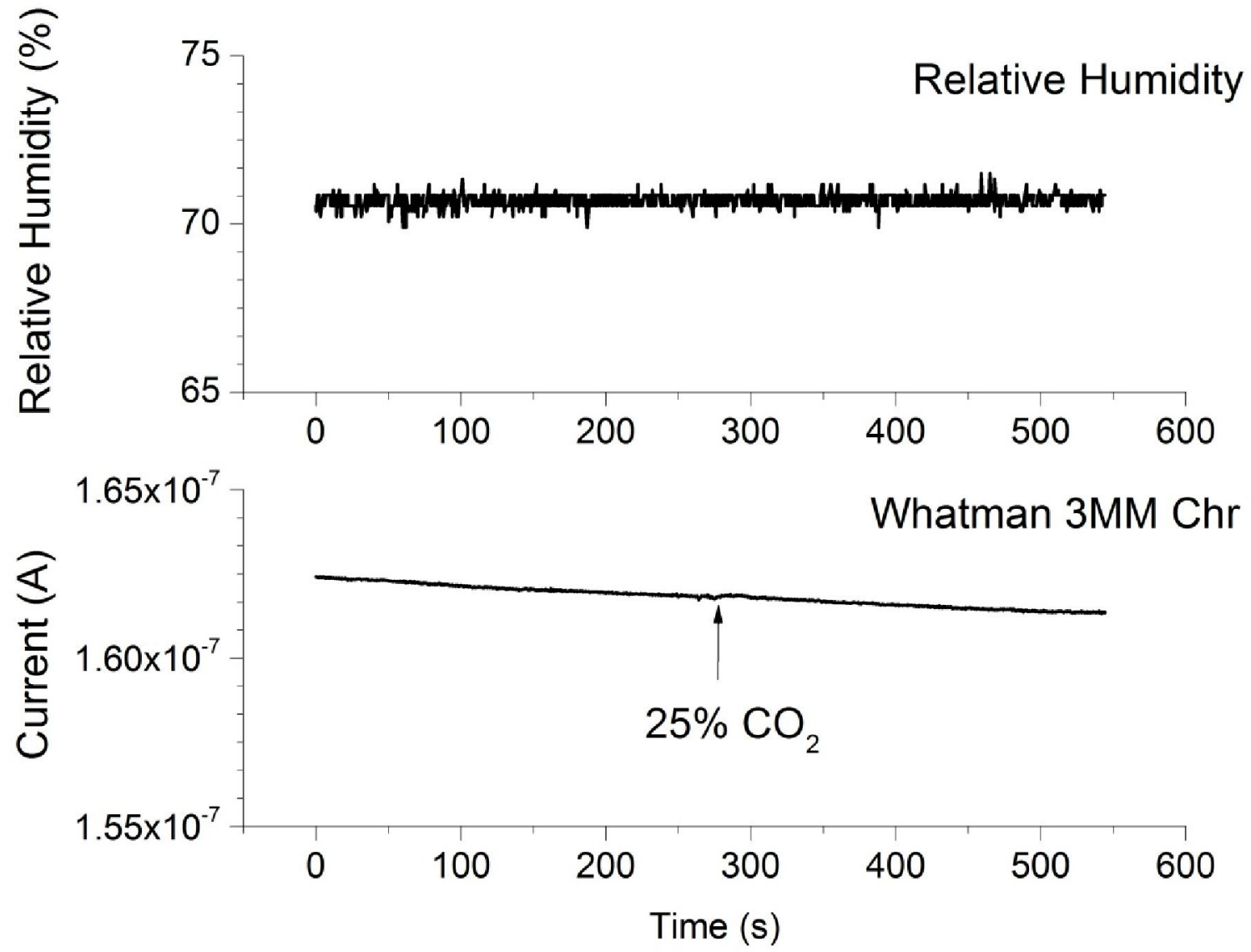

Figure S5. Response of the paper-based moisture sensor in the presence of $\mathrm{CO}_{2}$. In this experiment, the dry $\mathrm{N}_{2}$ stream is replaced with a $\mathrm{CO}_{2}$ stream using a three-way T-valve at $t=$ 270 s as indicated by the arrow in the figure. There was no increase in the sensor output when the sensor was exposed to $25 \% \mathrm{v} / \mathrm{v} \mathrm{CO}_{2}$. The $\mathrm{RH}$ inside the test chamber was kept constant at approx. $70 \%$ during the experiment. 


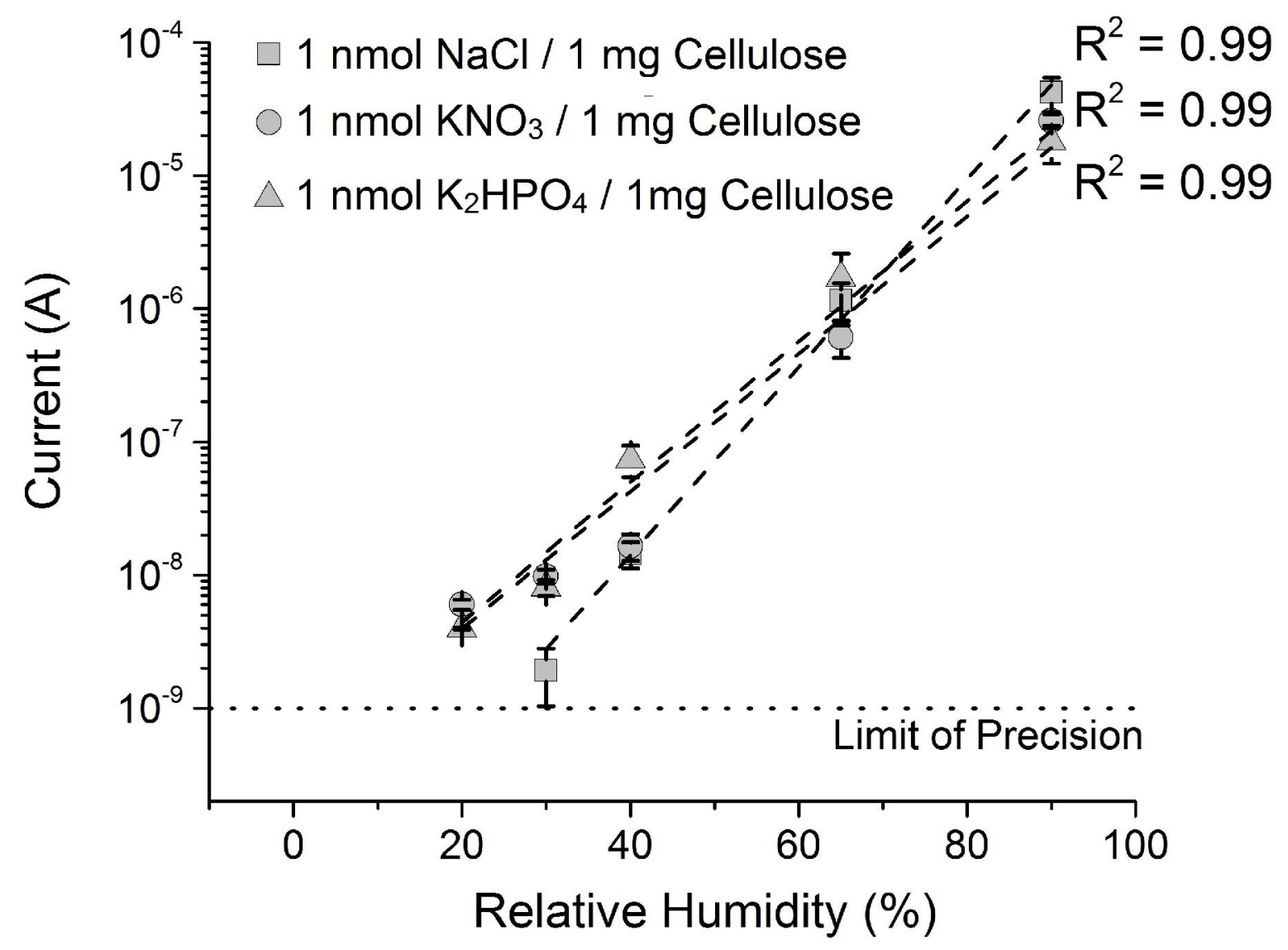

Figure S6. Characterization of paper-based moisture sensors with different salts $\left(\mathrm{KNO}_{3}\right.$, $\mathrm{K}_{2} \mathrm{HPO}_{4}, \mathrm{NaCl}$ ) at the same concentration over a range of $\mathrm{RH}$ of $0-90 \%$. We added $100 \mu \mathrm{L}$ of $1 \mathrm{mM}(100 \mathrm{nmol})$ aqueous solution of $\mathrm{KNO}_{3}, \mathrm{~K}_{2} \mathrm{HPO}_{4}, \mathrm{NaCl}$ on a paper sensor fabricated using Whatman 3MM Chr paper and evaporated the excess water in a conventional oven at $60{ }^{\circ} \mathrm{C}$ for one hour. To calculate the concentration of salt with respect to the amount of cellulose in paper, we cut a piece of Whatman 3MM Chr paper with the same size as the sensor (16 $\mathrm{mm}$ x $22 \mathrm{~mm}$ ) without the electrodes and weighed it on an analytical balance (100 mg). The addition of 100 nmol of salt resulted in a concentration of $1 \mathrm{nmol}$ of salt per $1 \mathrm{mg}$ of cellulose. We presume, small differences in the signal level are due to variations in hygroscopicity of the salts and solubility of the salts in water. The error bars represent standard deviations (SD). 


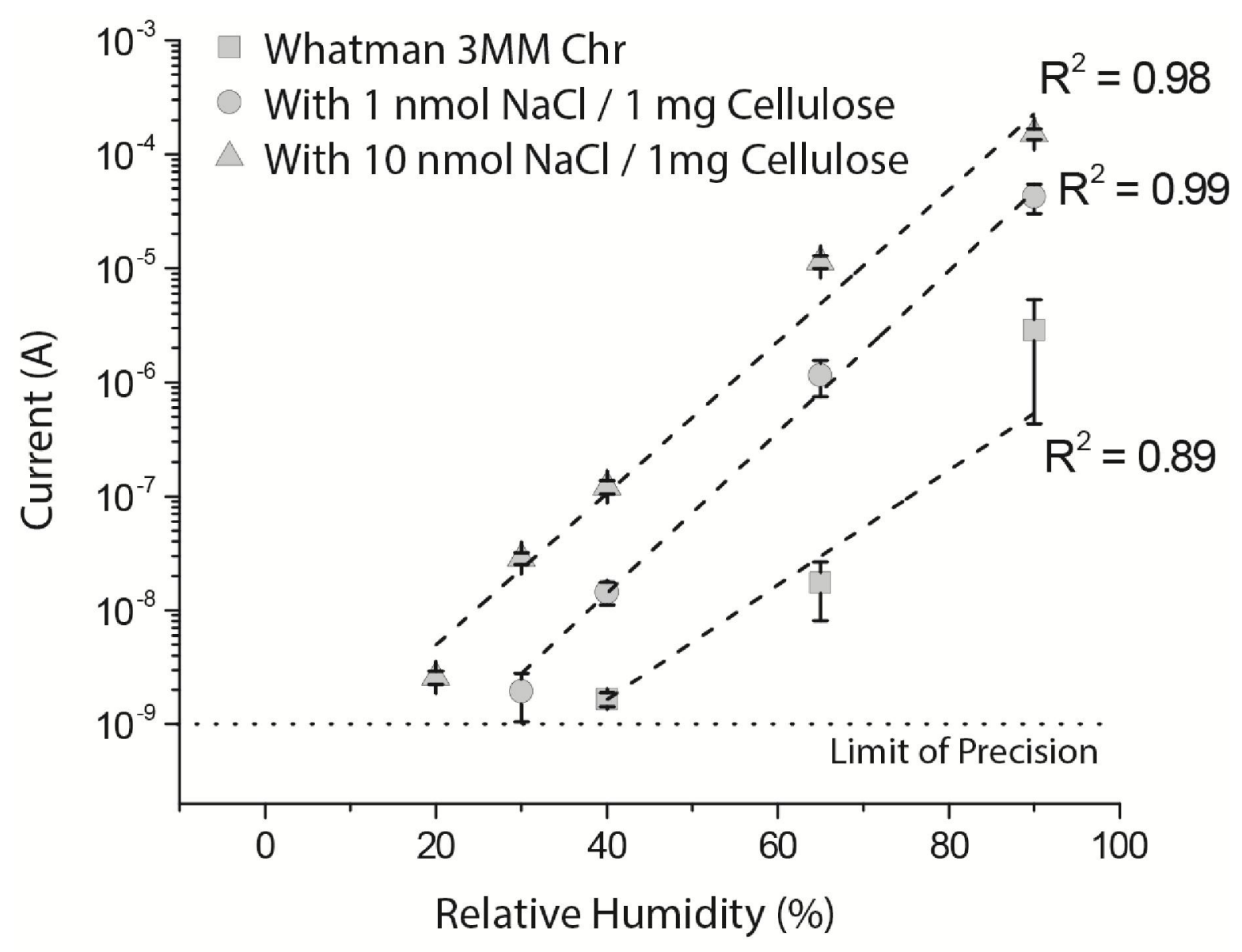

Figure S7. The effect of added $\mathrm{NaCl}$ on the electrical response of Whatman 3MM Chr paper at different salt concentrations. The error bars represent standard deviations (SD) 

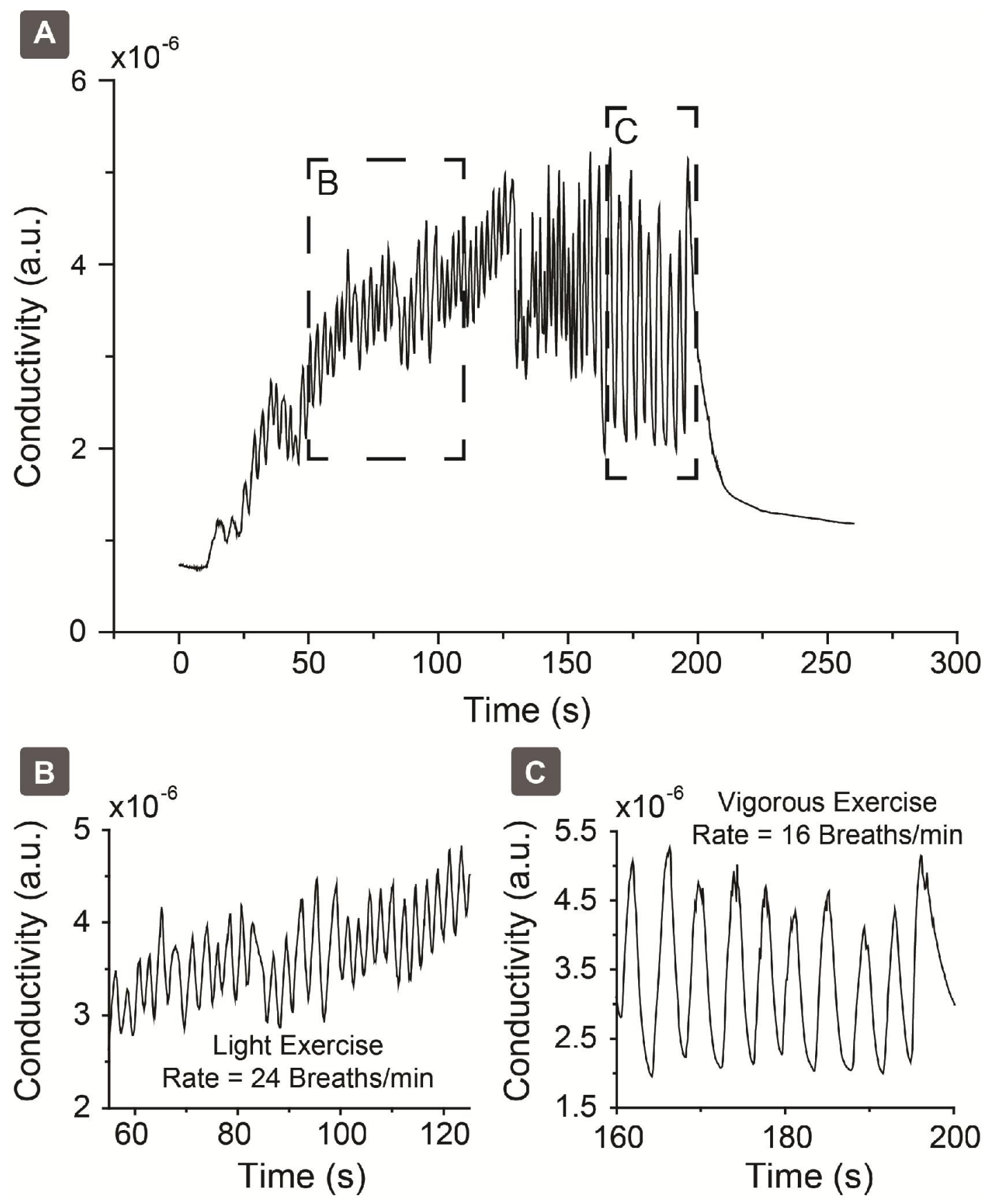

Figure S8. (A) Recorded respiratory signal during light (B) and vigorous (C) exercise. The subject in this experiment had a decrease in the rate of respiration from 24 breaths/min during light exercise to 16 breaths/min during vigorous exercise. The subject compensated for this reduction in the rate of respiration by taking deeper breaths. 


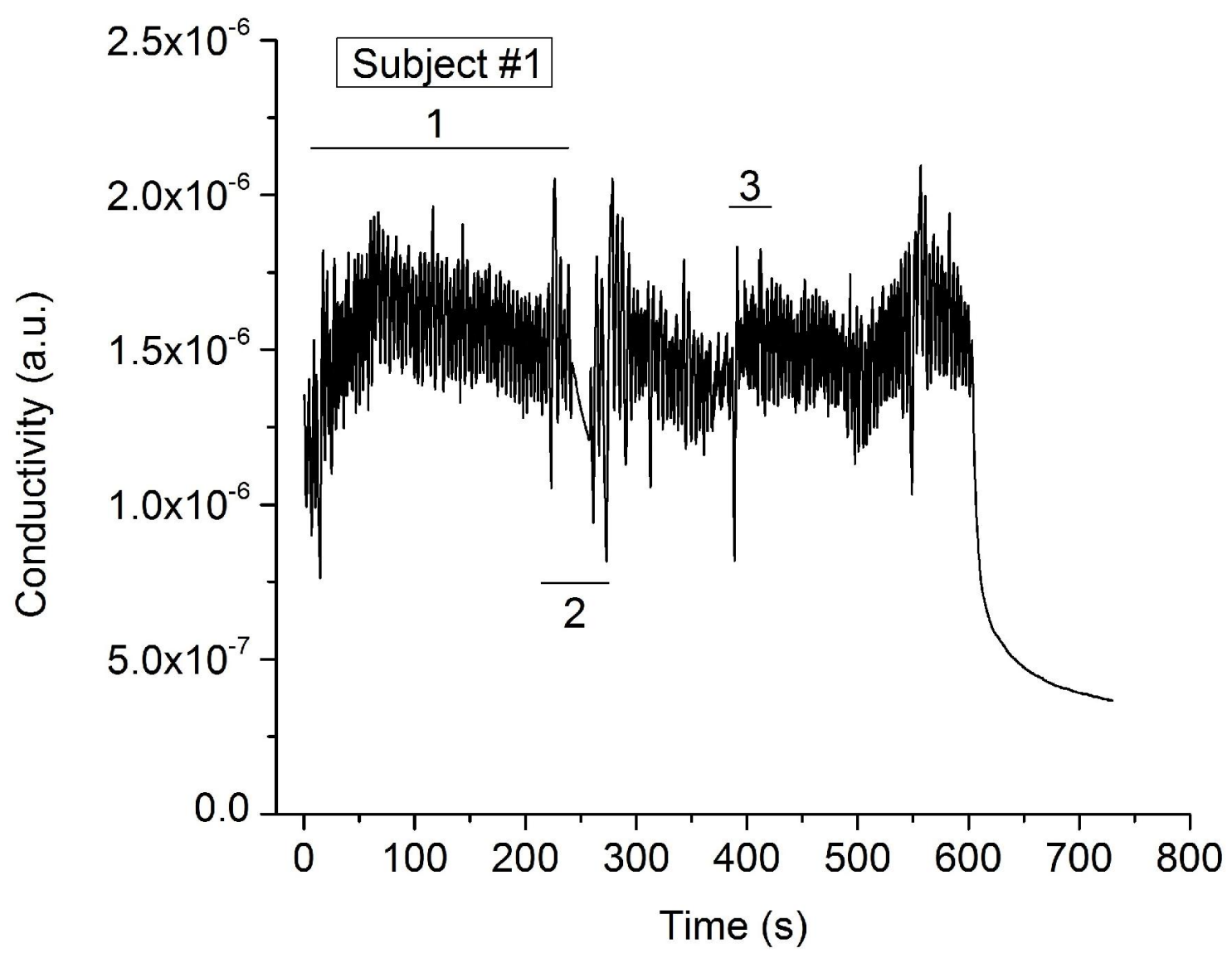

Figure S9. The resting respiratory activity of Subject \#1. (1) Indicates normal, (2) paused and, (3) fast and shallow breathing 


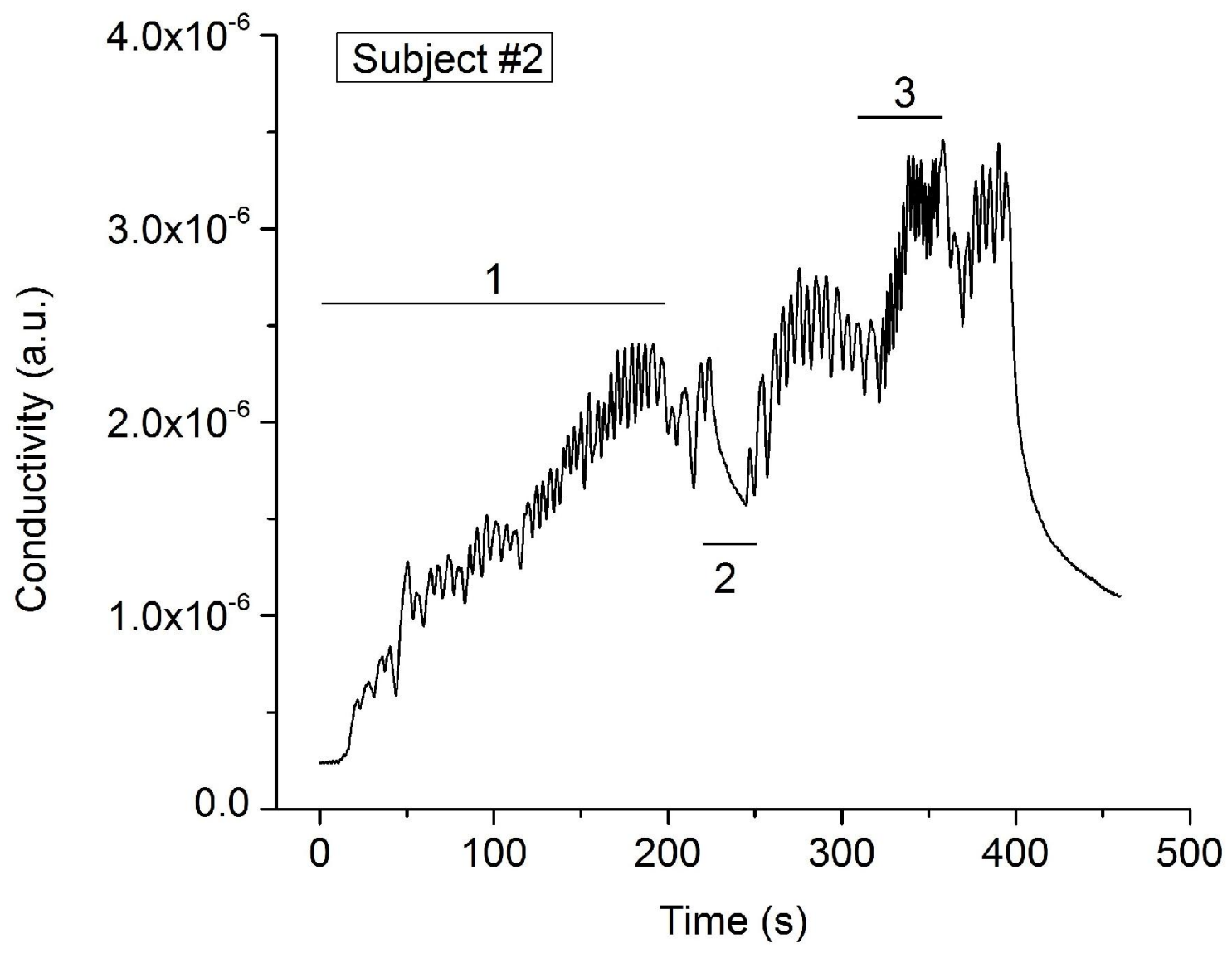

Figure S10. The resting respiratory activity of Subject \#2. (1) Indicates normal, (2) paused and, (3) fast and shallow breathing 


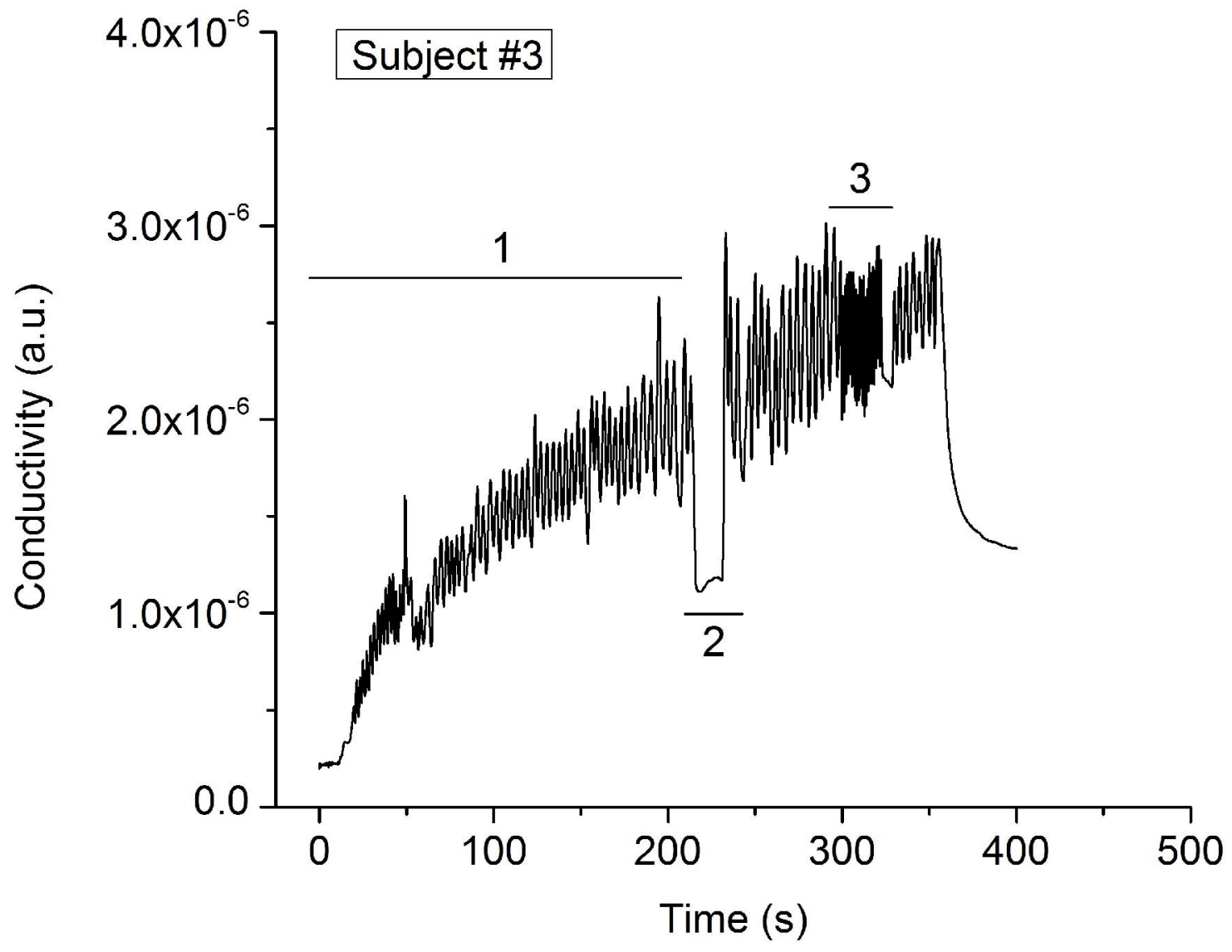

Figure S11. The resting respiratory activity of Subject \#3. (1) Indicates normal, (2) paused and, (3) fast and shallow breathing 


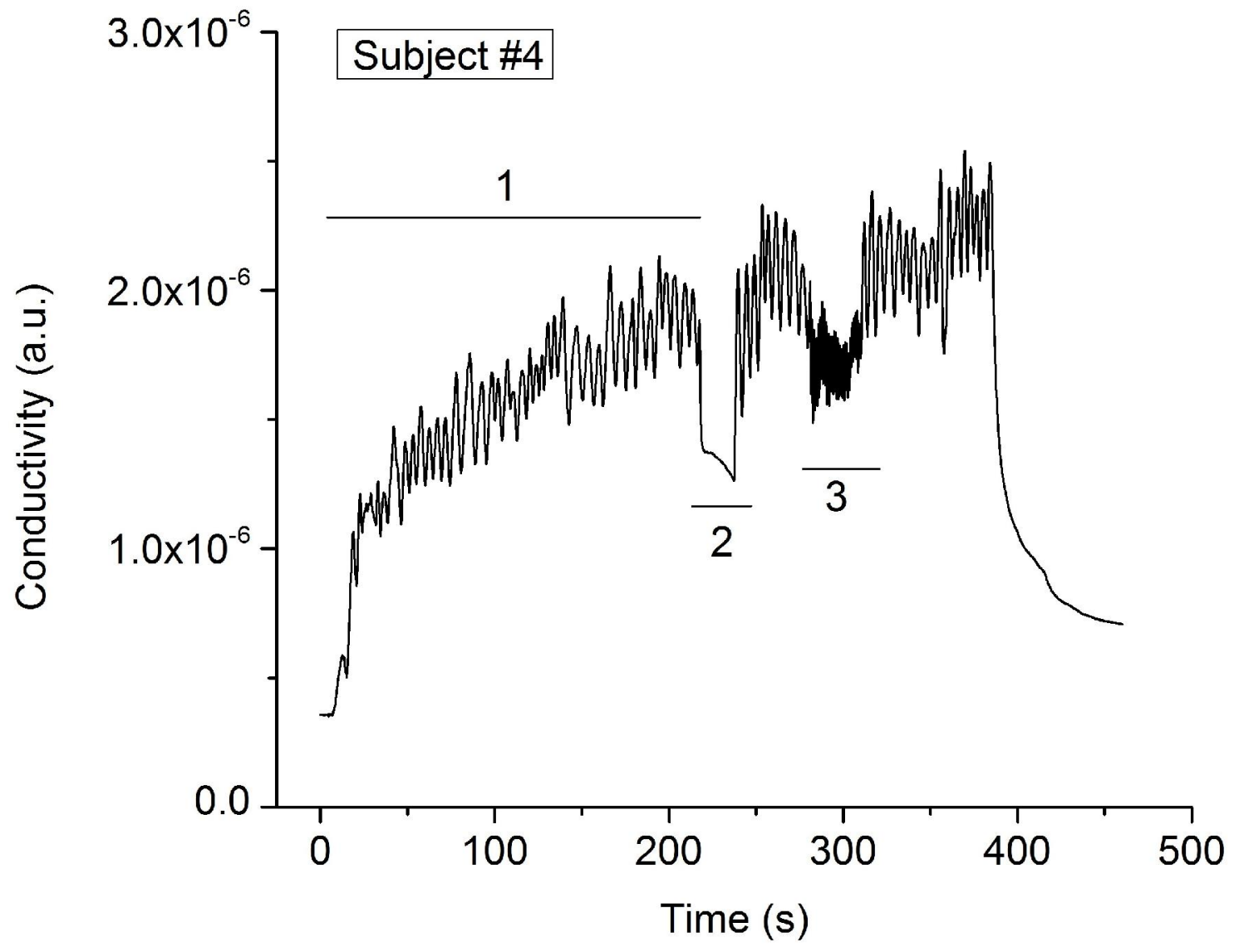

Figure S12. The resting respiratory activity of Subject \#4. (1) Indicates normal, (2) paused and, (3) fast and shallow breathing 


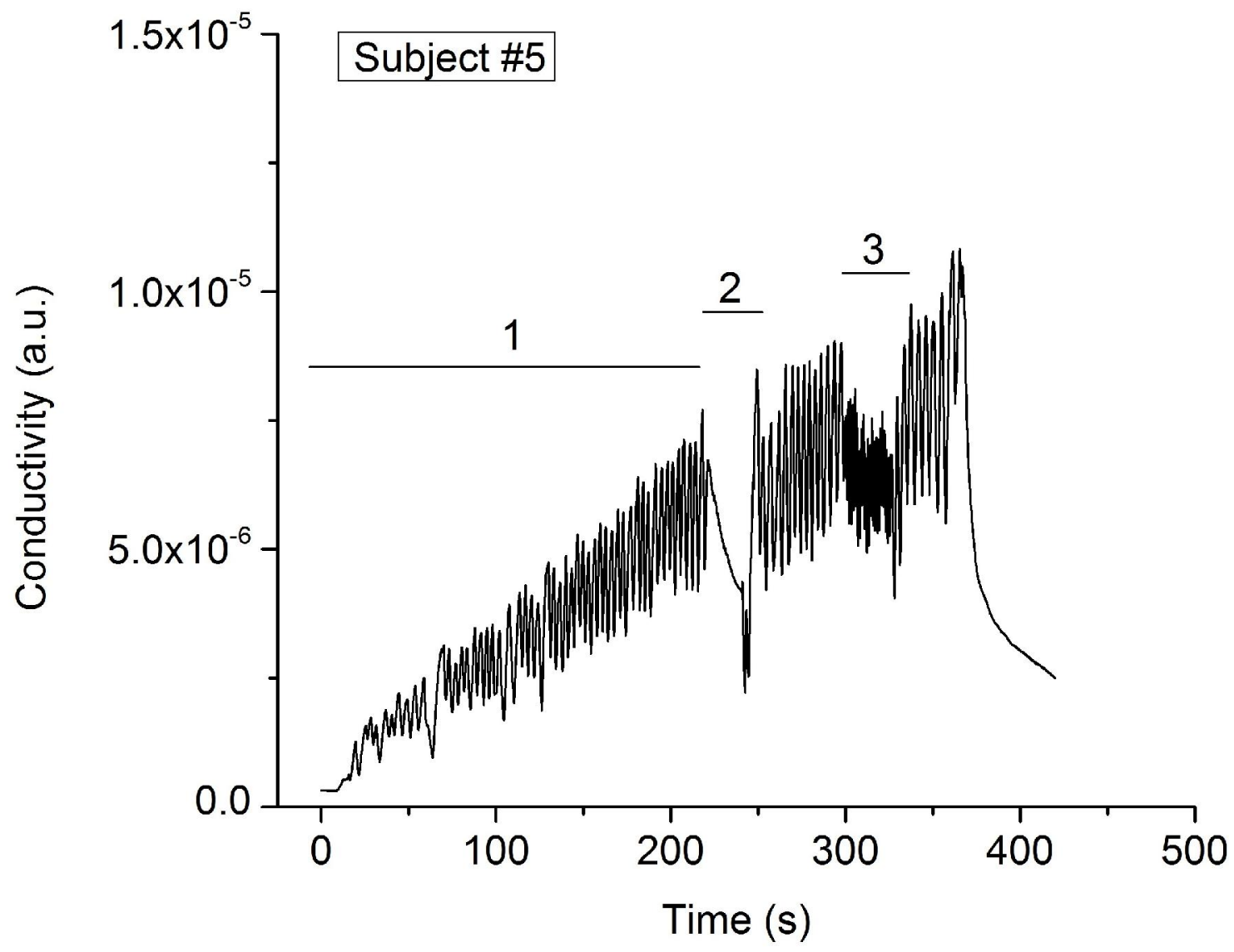

Figure S13. The resting respiratory activity of Subject \#5. (1) Indicates normal, (2) paused and, (3) fast and shallow breathing 


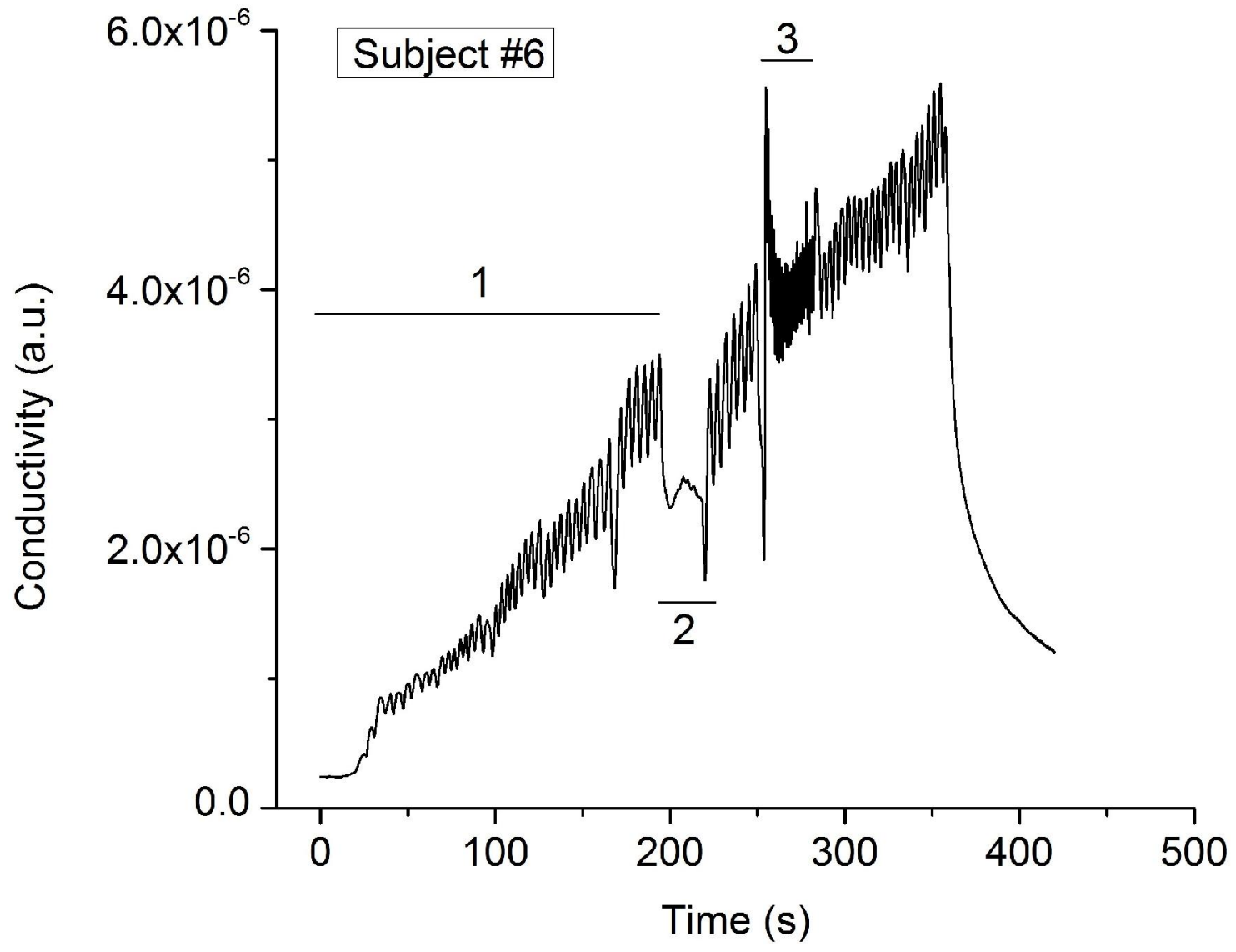

Figure S14. The resting respiratory activity of Subject \#6. (1) Indicates normal, (2) paused and, (3) fast and shallow breathing 


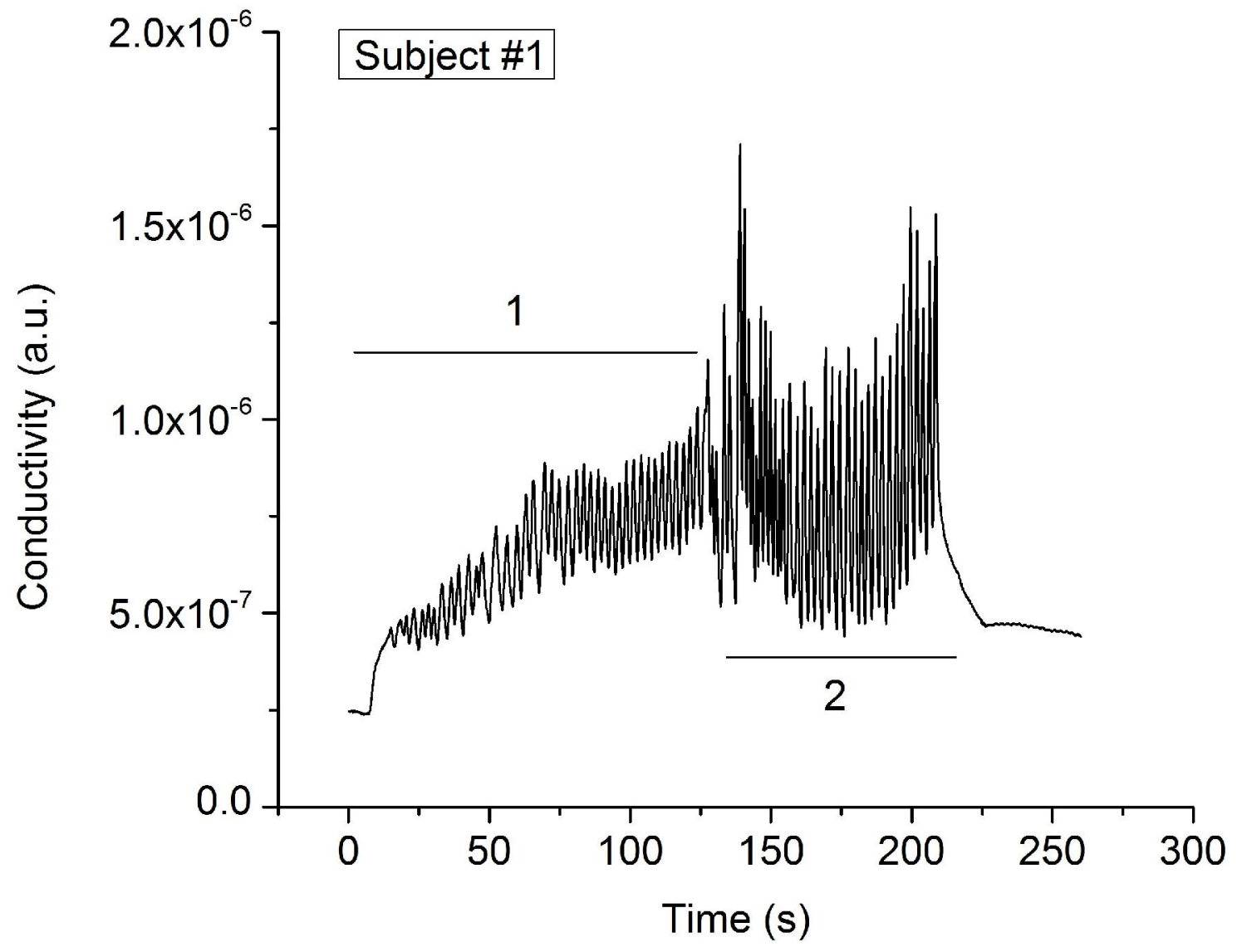

Figure S15. Recorded respiratory signal of Subject \#1 during light (1) and vigorous exercise (2). 


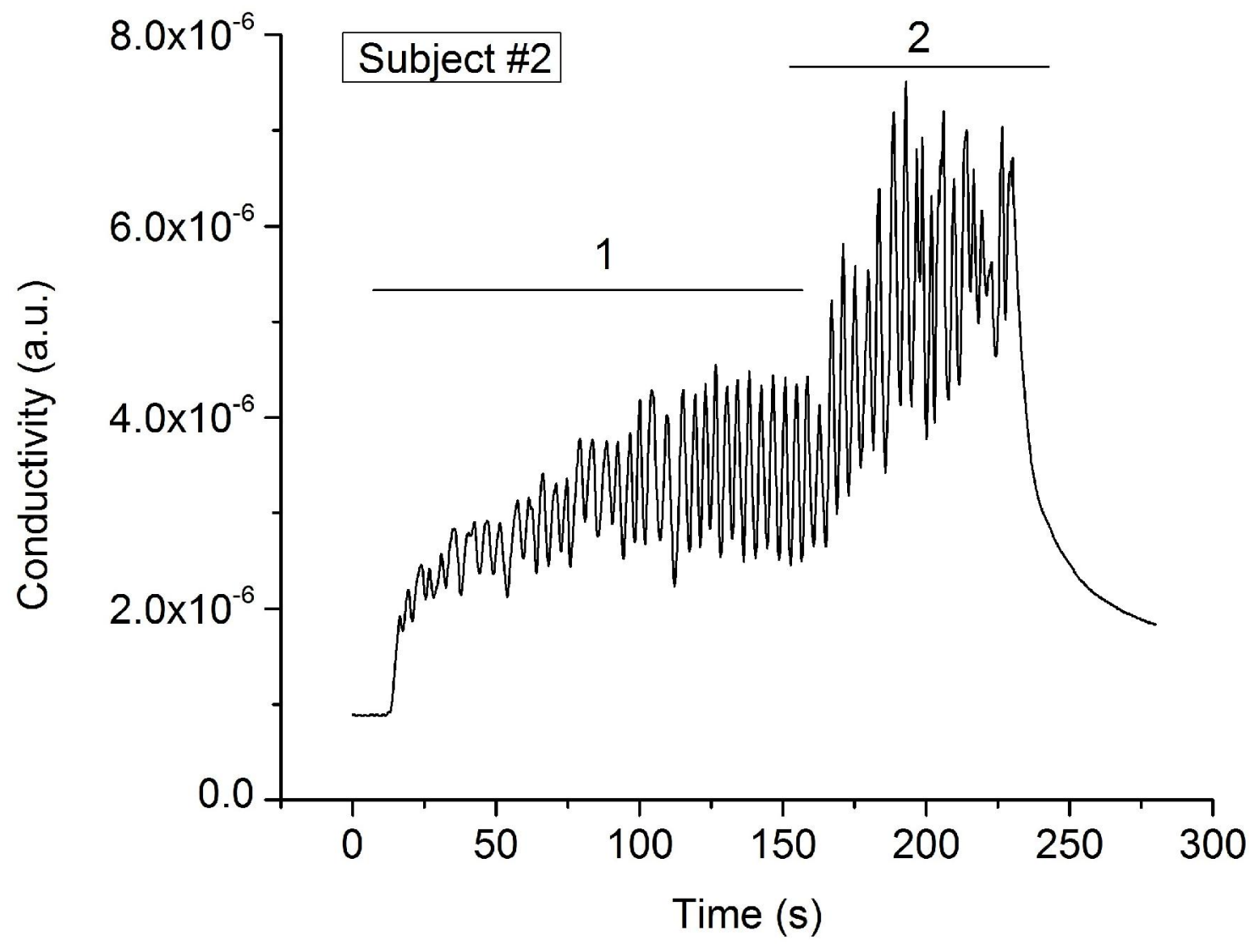

Figure S16. Recorded respiratory signal of Subject \#2 during light (1) and vigorous exercise (2). 


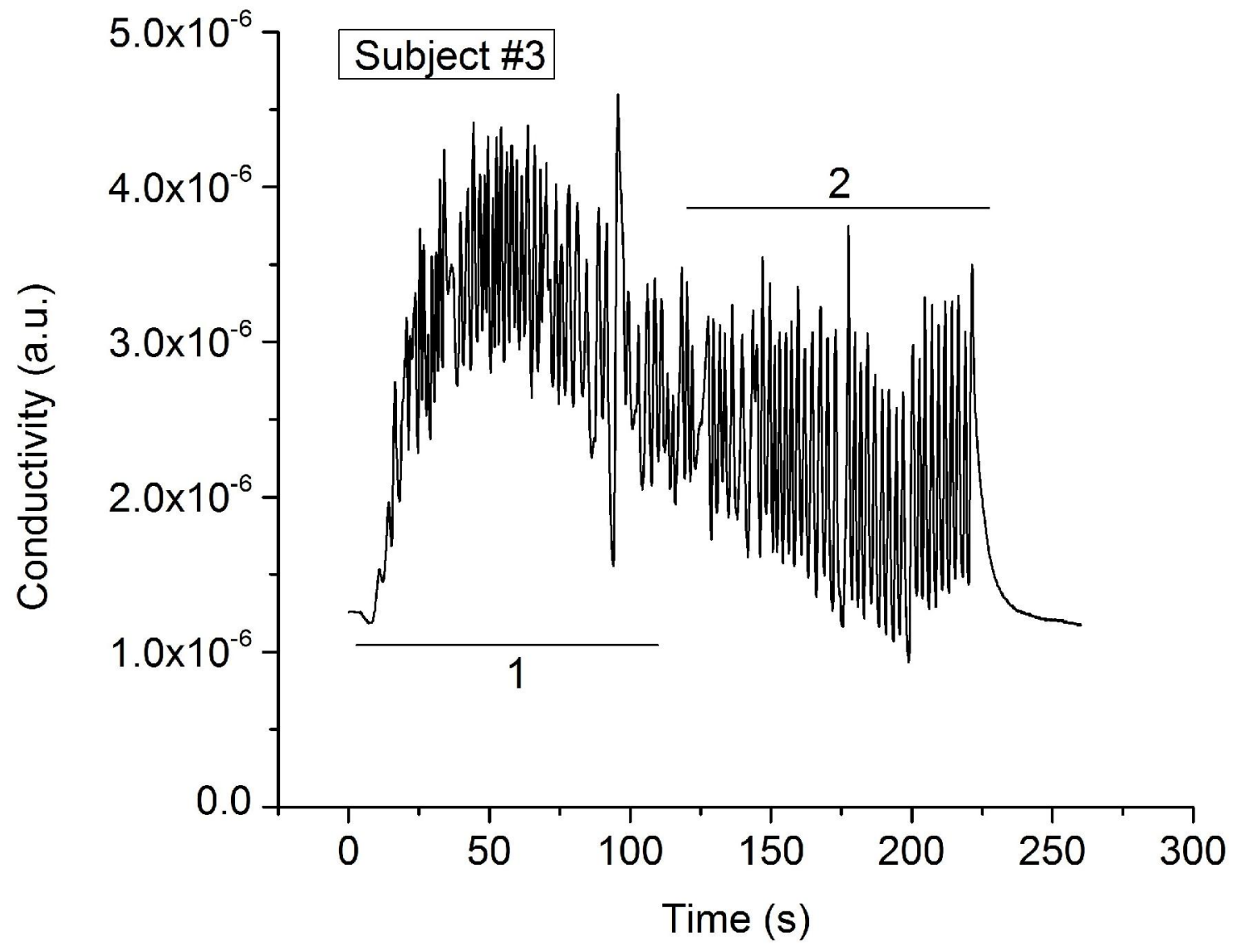

Figure S17. Recorded respiratory signal of Subject \#3 during light (1) and vigorous exercise (2). 


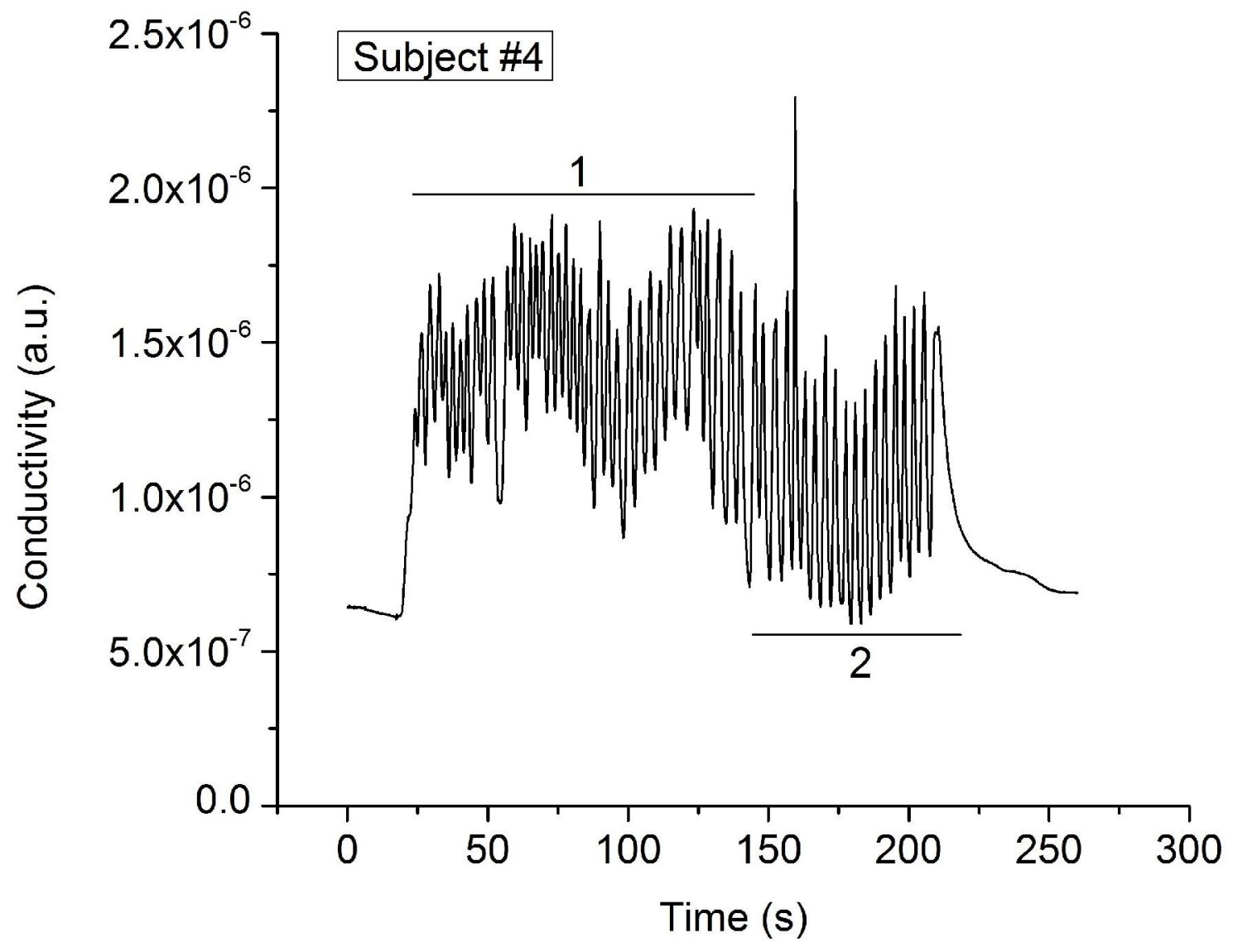

Figure S18. Recorded respiratory signal of Subject \#4 during light (1) and vigorous exercise (2). 


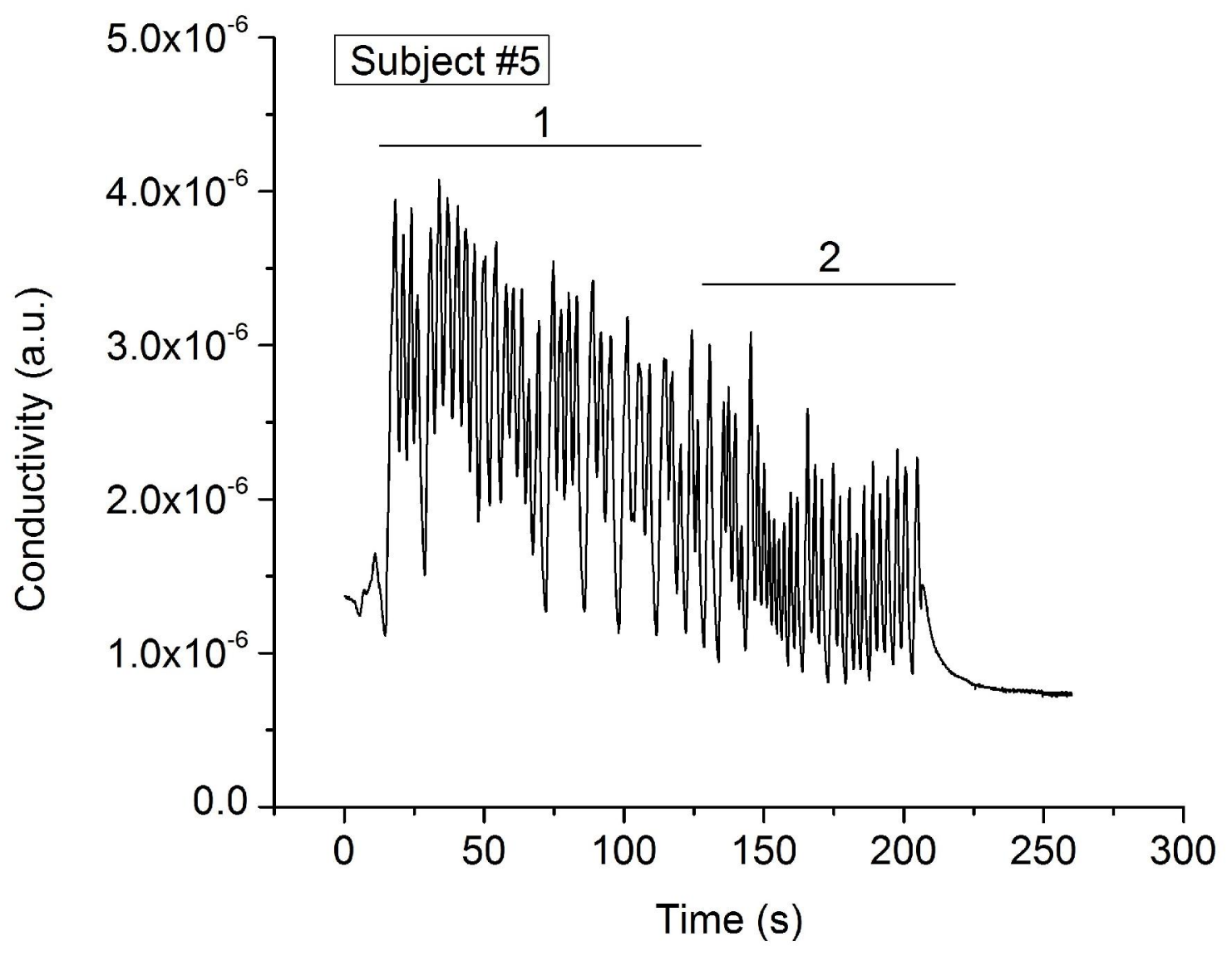

Figure S19. Recorded respiratory signal of Subject \#5 during light (1) and vigorous exercise (2). 


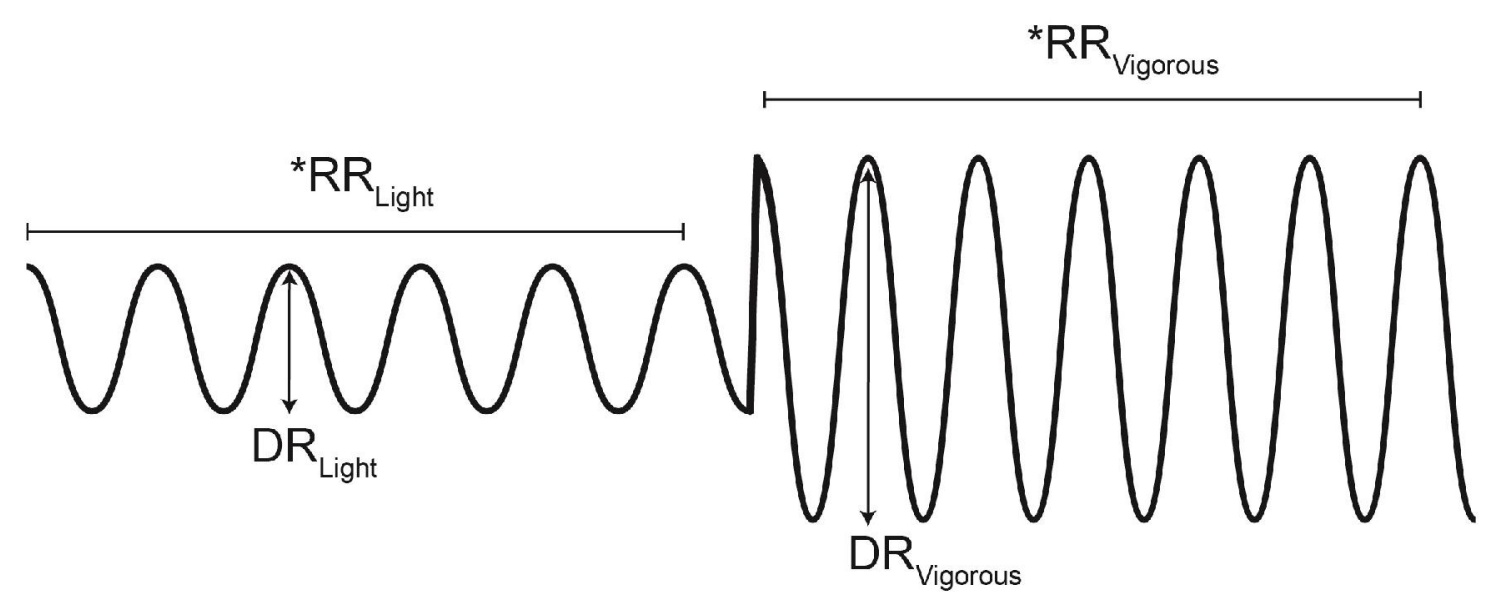

${ }^{*}$ Rate of respiration $(\mathrm{RR})$ is measured over 60 seconds

Figure S20. A conceptual waveform describing the parameters for calculating the empirical metric "Breathing Index" (BI); here, $\mathrm{RR}_{\text {vigorous }}$ is the rate of respiration during vigorous exercise, $\mathrm{RR}_{\mathrm{Light}}$ is the rate of respiration during light exercise, $\mathrm{DR}_{\mathrm{Vigorous}}$ is the peak-to-peak amplitude of the signal during vigorous exercise, and $\mathrm{DR}_{\mathrm{Light}}$, is the peak-to-peak amplitude of the signal during light exercise. BI is the product of the RR and DR. Since the baseline RR and DR of patients will vary between patients, we normalize the RR and DR values during vigorous exercise by the RR and DR values during light exercise for each patient, respectively. 